\title{
Performance Assessment of Linear State Estimators Using Synchrophasor Measurements
}

\author{
Styliani Sarri, Member, IEEE, Lorenzo Zanni, Member, IEEE, Miroslav Popovic, \\ Jean-Yves Le Boudec, Fellow, IEEE, and Mario Paolone, Senior Member, IEEE
}

\begin{abstract}
This paper aims to assess the performance of linear state estimation (SE) processes of power systems relying on synchrophasor measurements. The performance assessment is conducted with respect to two different families of SE algorithms, i.e., static ones represented by weighted least squares (WLS) and recursive ones represented by Kalman filter (KF). To this end, this paper firstly recalls the analytical formulation of linear WLS state estimator (LWLS-SE) and Discrete KF state estimator (DKF-SE). We formally quantify the differences in the performance of the two algorithms. The validation of this result, together with the comprehensive performance evaluation of the considered state estimators, is carried out using two case studies, representing distribution (IEEE 123-bus test feeder) and transmission (IEEE 39-bus test system) networks. As a further contribution, this paper validates the correctness of the most common process model adopted in DKF-SE of power systems.
\end{abstract}

Index Terms-Discrete Kalman filter (KF), linear state estimation (LSE), performance evaluation, phasor measurement unit (PMU), weighted least squares (WLS).

\section{INTRODUCTION}

$\mathbf{T}$ RADITIONAL power system state estimators rely on data coming from conventional and unsynchronized remote terminal units (RTUs). Therefore, they use the inherent nonlinear power flow equations since RTUs generally acquire powers and/or voltage/current magnitudes [1], [2]. As known, in case synchronized phasor measurements are available, there is the opportunity to take advantage of the linear relationship between the injected [3]-[5] and/or branch [4], [5] current measurements and the bus voltages (system state), in order to reformulate the state estimation (SE) problem in a linear way, i.e., linear SE (LSE). ${ }^{1}$

In this respect, the emerging availability of phasor measurement units (PMUs) allows acquiring accurate and timealigned phasors. The typical data streaming rates of PMUs

Manuscript received June 26, 2015; revised November 30, 2015; accepted December 1, 2015. This work was supported in part by the European Community's Seventh Framework Programme FP7-ICT-2011-8 through the C-DAX Project under Grant 318708 and in part by the NanoTera Swiss National Science Foundation Project S3-Grids. The Associate Editor coordinating the review process was Dr. Carlo Muscas.

The authors are with the École Polytechnique Fédérale de Lausanne, Lausanne CH-1015, Switzerland (e-mail: styliani.sarri@epfl.ch; lorenzo.zanni@epfl.ch; miroslav.popovic@epfl.ch; jean-yves.leboudec@ epfl.ch; mario.paolone@epfl.ch).

Color versions of one or more of the figures in this paper are available online at http://ieeexplore.ieee.org.

Digital Object Identifier 10.1109/TIM.2015.2510598

${ }^{1}$ In this paper, we target static SE, i.e., the estimation of voltage phasors at every bus, and not dynamic SE, i.e., the estimation of rotor angle and speed of generators [6]-[8]. are in the order of some tens of measurements per second [9], [10]. This technology is experiencing a fast evolution triggered by an increasing number of power system applications. PMUs already compose the backbone of wide-area monitoring systems in the context of transmission networks to which several real-time functionalities are connected. Typical examples are power system stability, interarea oscillations, voltage stability, relaying, and real-time SE. PMUs might represent fundamental monitoring tools even in the context of power distribution networks, where they can be used for various applications, such as SE [11], [12], loss of main [13], fault event monitoring [14], synchronous islanded operation [15] and for power quality monitoring [16]. With specific reference to the use of PMUs for SE in distribution networks, the recent literature has discussed the use of this technology not only from the methodological point of view [17] but also via dedicated realscale experimental setups [18], [19]. Also the adoption of low-cost dedicated hardware platforms is contributing to their potential massive use in distribution networks [20].

Due to their ability to estimate accurate phasor quantities and inherent real-time nature, PMUs today represent the most up-to-date metering technology for both transmission and distribution network operators interested in updating real-time control and protection functionalities. These functionalities may rely on subsecond LSE processes using PMU data [21]. It is worth reminding that typical refresh rates of the existing SCADA (Supervisory Control and Data Acquisition) SE processes are in the order of a few minutes/seconds. In this direction, the recent literature has discussed the use of PMU-based state estimators for several control applications [22], [23]. The effectiveness of these applications is largely improved if the variance of the estimated state is reduced.

Concerning the mathematical nature of the LSE methods, it is worth observing that they rely on a measurement model (see Section III) that has two main characteristics: 1) it is linear and 2) it is exact. Concerning the second of these properties, it does not hold in case classical power or pure-magnitude measurements are used in the SE. Indeed; in this case, the required linearization between available measurements and the system state is needed, involving a nontrivial impact on the measurement covariance assessment, the convergence of the SE process, and its accuracy. A straightforward consequence of properties 1) and 2) is that LSE mathematical characteristics are invariant with respect to the grid type, voltage, and topology. As a consequence, as already done by other 
contributions in the literature on this subject (see [24]-[27]), the assessment of the LSE performance can be done for both transmission and distribution networks (see Section IV). In this paper, we target SE for real-time applications, and therefore, we consider only synchronized phasor measurements and we do not use pseudo-measurements. Indeed, the latter, such as historical data, are traditionally used by network operators that target situational awareness at low refresh rates, i.e., in the range of minutes. In addition, the variances of pseudomeasurements are difficult to infer, and this can have a negative impact on the SE accuracy.

There are several important research works that have dealt with the LSE problem so far. The majority of them have used the weighted least squares (WLS) algorithm [3]-[5], [21]. Few of them have adopted the Kalman filter (KF) algorithm [28], [29]. However, to the best of our knowledge, none of the previous works has conducted a comparative performance assessment between WLS and KF-based SE algorithms. In this respect, the contributions of this paper are as follows:

1) a comparative assessment of the performance (accuracy and computational complexity) of the two considered families of linear state estimators ${ }^{2}$;

2) the numerical validation of the adopted discrete KF (DKF) process model, whose process noise covariance matrix is assessed via a heuristic method presented in $[29]^{3}$;

3) the formal and numerical proof of the better accuracy of DKF state estimators (DKF-SEs) over linear WLS state estimators (LWLS-SEs);

4) the computation performance analysis of the considered algorithms with respect to realistic networks: the IEEE 123-bus distribution test feeder [30] and the IEEE 39-bus transmission test system [31].

This paper has the following structure. Section II provides a literature review. In Section III the LSE problem is formulated with respect to the LWLS-SE and the DKF-SE. Concerning the DKF-SE, specific details are provided on the method used to assess the process noise covariance matrix. In the same section, we formally quantify the differences in the accuracy of the DKF-SE and the LWLS-SE. Section IV illustrates the numerical performance evaluation in terms of accuracy and computation time. The DKF process model validation together with the numerical proof of the better accuracy of the DKF-SE over the LWLS-SE is also given. Section V provides the final conclusion and remarks.

\section{LiterATURE REVIEW}

In this Section the most significant contributions to the literature on purely or partly PMU-based SE are given. Lu et al. [3] and Baran and Kelley [5] have proposed a three-phase (3-ph) current-based state estimator using the well-known WLS algorithm. In particular, Lu et al. [3] have also performed

\footnotetext{
${ }^{2}$ In this paper, we do not aim to assess the performance of the whole $\mathrm{SE}$ chain (i.e., the measurement devices, the telecom infrastructure, and the phasor data concentrator), as it has been done in [18].

${ }^{3}$ Although the method that allows assessing the DKF process noise covariance matrix has been presented in [29], its numerical validation is given in this paper for the first time.
}

a comparative analysis between classic nonlinear SE algorithms and an LWLS-SE using rectangular coordinates, and they have demonstrated that the latter has better performance in terms of computation time. In [4], a 3-ph linearized SE algorithm based on WLS has been proposed. The SE acquires bus voltage phasor measurements using PMUs, smart meter measurements, and pseudo-measurements. In [32], a two-stage state estimator has been presented. In the first stage, it uses a linear SE process relying on synchrophasor measurements provided by PMUs. In the second stage, the SE combines the estimated voltage phasors calculated in the first stage with conventional RTU data processed by a nonlinear state estimator. Pignati et al. [18], Jones et al. [21], and Sarri et al. [33] have used purely PMU-based LSEs implemented in real electrical networks. In particular, in [21], the LSE is based on the WLS algorithm with a refresh rate of 30 frames-persecond and is applied to a transmission network (subsection of Virginia Electric and Power Company's $500 \mathrm{kV}$ network). In [18] and [33], the LSE is based on the DKF algorithm with a refresh rate of 50 frames-per-second and is applied to the distribution networks of the Swiss Federal Institute of Technology of Lausanne campus and the BML 2.10 feeder of Alliander in the Netherlands, respectively. Nuqui and Phadke [34] have proposed a hybrid state estimator with a measurement model that combines the estimated state from the classical state estimator with the direct state measured by the PMUs, whereas [35] is a review paper on tracking and dynamic SE techniques. Bian et al. [36] have presented an approach defined as state tracking with correlated predictionmeasurement errors that can account for possible abrupt state change. Glavic and Van Cutsem [37] have presented a method to track the changing system state by taking advantage of the synchronized phasor measurements available at much higher rate than classical SE measurements, but in a limited number. They solve the resulting unobservability by complementing the PMU data with pseudo-measurements and zero-injection information. Finally, Muscas et al. [38] have presented a twostep procedure in order to estimate the state of a large-scale distribution network by dividing it into subareas. For each area, they first use a dedicated estimator, and then, data provided by local estimators are further processed to refine the knowledge of the operating conditions of the network. The estimates are improved by evaluating correlations that arise in the first step and considering them in the second step. The overall execution time of the process is significantly reduced since the two-step procedure is performed in a decentralized way with parallel processing.

\section{Problem Formulation}

\section{A. Linear Weighted Least Squares State Estimator}

This section summarizes the analytical formulation of the LWLS-SE for the case of generic 3-ph systems.

The system state for a network with $n$ buses $\mathbf{x} \in \mathbb{R}^{S}$ $(S=3 \cdot 2 n)$ is represented by the real and imaginary parts of the voltage phasors

$$
\mathbf{x}=\left[\mathbf{V}_{1, r e}^{a, b, c}, \ldots, \mathbf{V}_{n, r e}^{a, b, c}, \mathbf{V}_{1, i m}^{a, b, c}, \ldots, \mathbf{V}_{n, i m}^{a, b, c}\right]^{T}
$$


where

$$
\begin{aligned}
\mathbf{V}_{i, r e}^{a, b, c} & =\left[V_{i, r e}^{a}, V_{i, r e}^{b}, V_{i, r e}^{c}\right] \\
\mathbf{V}_{i, i m}^{a, b, c} & =\left[V_{i, i m}^{a}, V_{i, i m}^{b}, V_{i, i m}^{c}\right]
\end{aligned}
$$

are the 3-ph real and imaginary parts of the voltage phasor at bus $i(i=1, \ldots, n)$, respectively. It is worth mentioning that state estimators using branch currents as state variables have been proposed, for instance, in [5]. However, their performance is comparable with voltage-based state estimators as presented in [39]. For the sake of space, we limit our analysis on the latter category since similar results can be obtained for the former.

We assume that the measurements come only from PMUs installed in $d$ network buses and that the measurement set is composed of:

1) $3 d$ phase-to-ground voltage phasors;

2) $3 d$ injected current phasors.

As known, the measurement set can be expanded to include branch currents and pseudo-measurements, but in this paper, we limit our study to bus voltage and injected current phasors.

Therefore, the measurement set $\mathbf{z} \in \mathbb{R}^{D}(D=2 \cdot(3 d+3 d))$ is equal to

$$
\mathbf{z}=\left[\mathbf{z}_{V}, \mathbf{z}_{I}\right]^{T}
$$

where

$$
\begin{aligned}
\mathbf{z}_{V} & =\left[\mathbf{V}_{1, r e}^{a, b, c}, \ldots, \mathbf{V}_{d, r e}^{a, b, c}, \mathbf{V}_{1, i m}^{a, b, c}, \ldots, \mathbf{V}_{d, i m}^{a, b, c}\right] \\
\mathbf{z}_{I} & =\left[\mathbf{I}_{1, r e}^{a, b, c}, \ldots, \mathbf{I}_{d, r e}^{a, b, c}, \mathbf{I}_{1, i m}^{a, b, c}, \ldots, \mathbf{I}_{d, i m}^{a, b, c}\right]
\end{aligned}
$$

The equation that relates the measurements with the system state is

$$
\mathbf{z}=\mathbf{H x}+\boldsymbol{\varepsilon}
$$

where $\mathbf{H}$ is a $D \times S$ matrix that represents the link between the state and the measurements ${ }^{4}$ for the case of null measurement noise and $\varepsilon$ is the measurement noise. At this point, it is important to recall that $\mathbf{H}$ does not represent a linear approximation of the measurement model, since it corresponds to the exact link between measurements and state. As stated in Section I, the exactness of the measurement model expressed by (5) is valid irrespectively of the targeted network. The only property that should hold is that matrix $\mathbf{H}$ needs to be characterized by a full rank. Therefore, if the error $\varepsilon$ is associated with the true metrological characteristics of measurement devices used in transmission and distribution networks, it is possible to carry out an LSE performance assessment for both of these kinds of networks (see Section IV).

Assuming that the measurement noise is white and Gaussian and the measurement errors are independent, we get

$$
\begin{array}{r}
p(\boldsymbol{\varepsilon}) \sim N\left(0, \mathbf{R}_{k}\right) \\
\mathbf{R}_{k}=\operatorname{diag}\left(\boldsymbol{\sigma}_{\boldsymbol{\varepsilon}, 1}^{2}, \ldots, \boldsymbol{\sigma}_{\boldsymbol{\varepsilon}, D}^{2}\right)
\end{array}
$$

\footnotetext{
${ }^{4}$ The observability analysis is beyond the scope of this paper. However, the locations of the measurements have been selected in such a way to obtain the matrix $\mathbf{H}$ of full rank, a sufficient condition that makes the grid observable [40], [41].
}

where $k$ is the time step index, $\mathbf{R}_{k}$ is the so-called measurement noise covariance matrix, and $\boldsymbol{\sigma}_{\varepsilon, j}(j=1, \ldots, D)$ is the standard deviation of the $j^{\text {th }}$ measurement. Therefore, $\mathbf{R}_{k}$ represents the accuracies of the measurement devices. Note that the normality of PMU errors is based on experimental evidences of error distributions of actual PMUs [42].

At this point, it is important to mention that, in principle, as analytically described in [43] and [44], $\mathbf{R}_{k}$ can be a full matrix, since it is generally inappropriate to consider the measurement errors independent unless this hypothesis is properly justified. In our case, based on the observations that are mentioned in the following, we can neglect the correlations between different PMU measurements.

1) Measurement values given by different devices can be reasonably considered independent [43].

2) Measurements come only from PMUs, i.e., we do not use any conventional measurement (e.g., power flows and power injections) or pseudo-measurements.

3) The measurements of voltage and current amplitudes in the same PMU can be usually considered uncorrelated [43].

4) As demonstrated in [43], neglecting PMU correlations (both in amplitude and phase) in the estimator model does not lead to a significant decrease of the SE quality.

5) Voltage and current phasors are measured separately, per phase, i.e., we do not assume to use any 3-ph multifunction meter [44].

6) Based on the nomenclature and definitions given in [44], we use only independent Gaussian-distributed measured data and not dependent processed measurements.

7) The sensors cross-talk interferences are negligible and we use a unique sensor per measured quantity (i.e., voltage/current).

In view of the above, we have assumed to have Gaussiandistributed and independent measurement errors, and therefore, $\mathbf{R}_{k}$ is diagonal.

The real and imaginary parts of the 3-ph injected current phasors are

$$
\begin{aligned}
& I_{i, r e}^{p}=\sum_{h=1}^{n} \sum_{m=1}^{3}\left[G_{i h}^{p m} V_{h, r}^{m}-B_{i h}^{p m} V_{h, i m}^{m}\right] \\
& I_{i, i m}^{p}=\sum_{h=1}^{n} \sum_{m=1}^{3}\left[G_{i h}^{p m} V_{h, i m}^{m}+B_{i h}^{p m} V_{h, r}^{m}\right]
\end{aligned}
$$

where $i$ and $h$ are the bus indexes, $p$ and $m$ are the phase indexes, and $G$ and $B$ are respectively the real and imaginary parts of the admittance matrix elements.

In this respect, $\mathbf{H}$ is equal to

$$
\mathbf{H}=\left[\begin{array}{l}
\mathbf{H}_{\mathbf{V}} \\
\mathbf{H}_{\mathbf{I}}
\end{array}\right]
$$

where $\mathbf{H}_{\mathbf{V}}$ is the part related to the bus voltage measurements, consisting of ones and zeros and directly inferred from (5), whereas $\mathbf{H}_{\mathbf{I}}$ is the part related to the injected current measurements and can be derived in a straightforward way from (8) and (9)

$$
\mathbf{H}_{\mathbf{I}}=\left[\begin{array}{cc}
G_{i h}^{p m} & -B_{i h}^{p m} \\
B_{i h}^{p m} & G_{i h}^{p m}
\end{array}\right] .
$$


The LWLS-SE maximizes the likelihood that, as known, is equivalent to minimizing the following objective function:

$$
J(\mathbf{x})=\sum_{j=1}^{D} \frac{\left(z_{j}-\sum_{r=1}^{S} H_{j r} x_{r}\right)^{2}}{R_{j j}} .
$$

Then, we calculate the so-called gain matrix for the current time step $k$

$$
\mathbf{G}_{k}=\mathbf{H}^{T} \mathbf{R}_{k}^{-1} \mathbf{H} .
$$

The estimated system state is equal to

$$
\widehat{\mathbf{x}}_{\mathrm{LWLS}, k}=\mathbf{G}_{k}^{-1} \mathbf{H}^{T} \mathbf{R}_{k}^{-1} \mathbf{z}_{k} \text {. }
$$

\section{B. Discrete Kalman Filter State Estimator}

The DKF-SE algorithm, assuming that there are no control inputs, is described by the following set of equations [28], $[45]^{5}$ :

$$
\begin{aligned}
\mathbf{x}_{k} & =\mathbf{x}_{k-1}+\mathbf{w}_{k-1} \\
\mathbf{z}_{k} & =\mathbf{H x}_{k}+\boldsymbol{\varepsilon}_{k}
\end{aligned}
$$

where $\mathbf{w} \in \mathbb{R}^{S}$ represents the process noise, assumed white Gaussian and independent of $\boldsymbol{\varepsilon}$.

Equation (15) represents an Auto Regressive Integrated Moving Average-ARIMA $(0,1,0)$ process that is a special case of the generic well-known $\mathrm{KF}$ process model $\mathbf{x}_{k}=$ $\mathbf{A} \mathbf{x}_{k-1}+\mathbf{B} \mathbf{u}_{k-1}+\mathbf{w}_{k-1}$. For the case of null control inputs and process noise, $\mathbf{A}$ is the matrix that links the system state at time step $k-1$ with the one at the current time step $k$ and $\mathbf{u}_{k-1}$ represents the set of control variables at time step $k-1$. For the case of null process noise, $\mathbf{B}$ is the matrix that links the control variables at time step $k-1$ with the state at the current time step $k$. Hence, the process model of this paper corresponds to $\mathbf{A}=\mathbf{I}$ and $\mathbf{B}=\mathbf{0}$, the latter being because we have no control inputs. This specific choice has also been used by Debs and Larson [45] and is motivated by the principle of parsimony (there is no parameter to estimate in A) and by the fact that with high-resolution measurements, it is generally a good idea to predict the next state by the current one. In Section IV-C, for the first time in the literature on power systems, we evaluate the adequacy of this model for the specific case of SE.

The DKF algorithm is characterized into two covariance matrices: 1) the measurement noise covariance matrix $\mathbf{R}_{k}$ given in (7) and 2) the process noise covariance matrix $\mathbf{Q}_{k}$ that represents the uncertainties introduced by the process model to predict the next system state

$$
p(\mathbf{w}) \sim N\left(0, \mathbf{Q}_{k}\right) .
$$

The matrix $\mathbf{Q}_{k}$ has a significant impact on the $\mathrm{SE}$ results. Therefore, Section III-C describes analytically the procedure that has been adopted in order to assess $\mathbf{Q}_{k}$. Note the dependency on time, witnessed by the index $k$.

\footnotetext{
5 It is worth noting that (16) is similar to (5). However, it is given again to describe the complete DKF-SE. Indeed, in (16), the measurement model (5) at time step $k$ is combined with a process model that uses information available at time step $k-1$.
}

As known [46], [47], DKF consists of two different parts, the so-called time update/prediction and the measurement update/estimation. If we define the true state as $\mathbf{x}_{k}$, the predicted state as $\widetilde{\mathbf{x}}_{k}$, and the estimated state as $\widehat{\mathbf{x}}_{k}$, the following errors are derived:

$$
\begin{aligned}
\widetilde{\mathbf{e}}_{k} & =\mathbf{x}_{k}-\widetilde{\mathbf{x}}_{k} \\
\mathbf{e}_{k} & =\mathbf{x}_{k}-\widehat{\mathbf{x}}_{k} .
\end{aligned}
$$

The prediction error covariance matrix is then

$$
\widetilde{\mathbf{P}}_{k}=\mathbb{E}\left(\widetilde{\mathbf{e}}_{k} \widetilde{\mathbf{e}}_{k}^{T}\right)
$$

and the estimation error covariance matrix is

$$
\mathbf{P}_{k}=\mathbb{E}\left(\mathbf{e}_{k} \mathbf{e}_{k}^{T}\right)
$$

where $\mathbb{E}()$ denotes the expected value.

The estimated state is then obtained in the following way.

1) The time update/prediction is given by

$$
\begin{aligned}
\widetilde{\mathbf{x}}_{k} & =\widehat{\mathbf{x}}_{k-1} \\
\widetilde{\mathbf{P}}_{k} & =\mathbf{P}_{k-1}+\mathbf{Q}_{k-1} .
\end{aligned}
$$

2) The measurement update/estimation is given by

$$
\begin{aligned}
\mathbf{K}_{k} & =\widetilde{\mathbf{P}}_{k} \mathbf{H}^{T}\left(\mathbf{H} \widetilde{\mathbf{P}}_{k} \mathbf{H}^{T}+\mathbf{R}_{k}\right)^{-1} \\
\widehat{\mathbf{x}}_{k} & =\widetilde{\mathbf{x}}_{k}+\mathbf{K}_{k}\left(\mathbf{z}_{k}-\mathbf{H} \widetilde{\mathbf{x}}_{k}\right) \\
\mathbf{P}_{k} & =\left(\mathbf{I}-\mathbf{K}_{k} \mathbf{H}\right) \widetilde{\mathbf{P}}_{k}
\end{aligned}
$$

where $\mathbf{K}$ is the so-called Kalman Gain.

\section{Assessment of the Process Noise Covariance Matrix}

In the literature dealing with power systems SE using DKF, the values of $\mathbf{Q}_{k}$ are, usually, arbitrarily selected although, in principle, they can be computed if the process is known [35], [48]. Since it can significantly influence the DKF accuracy, an appropriate assessment of this matrix is of fundamental importance for the maximization of the DKF-SE accuracy. Mehra [49] and Najim [50] have described a classic method to estimate the covariance matrices of both the process and measurement noises that uses a three-step procedure. More recently, Odelson et al. [51] have proposed a one-step procedure that consists in solving an optimization problem that imposes semidefinite constraints on the covariance matrices. Hence, it guarantees the uniqueness of the estimated covariances and positive semidefinite covariance estimates. These methods work only in steady-state conditions and are computationally expensive. Therefore, we have decided to use a heuristic method proposed in [29] that is effective in terms of estimation accuracy and suitable for real-time applications. It is based on the fact that $\mathbf{Q}_{k}$ is not supposed to change dramatically if its update rate is high enough compared with the dynamics that take place in the network. In this respect, we make reference to PMU-based state estimators characterized by refresh rates that can reach tens of frames-per-second.

In what follows, we recall the analytical formulation of the method for the assessment of the process noise covariance matrix $\mathbf{Q}_{k}$ proposed in [29]. At time step $k$, the estimation of $\mathbf{Q}_{k}$ is performed using the last $N$ estimated states. The procedure is as follows.

1) Compute the vectors $\mathbf{y}_{c} \in \mathbb{R}^{S}(c=1, \ldots, N)$ as

$$
\mathbf{y}_{c}=\widehat{\mathbf{x}}_{k-c}-\widehat{\mathbf{x}}_{k-N-1} .
$$


2) The $r^{\text {th }}$ element of the vector $\sigma_{\mathbf{y}}^{2} \in \mathbb{R}^{S}$ is the sample variance

$$
\sigma_{\mathbf{y}}^{2}(r)=\operatorname{var}\left[\mathbf{y}_{0}(r), \ldots, \mathbf{y}_{N-1}(r)\right] .
$$

3) $\mathbf{Q}_{k}$ is the diagonal matrix whose elements are $\sigma_{\mathbf{y}}^{2}$

$$
\mathbf{Q}_{k}=\operatorname{diag}\left(\boldsymbol{\sigma}_{\mathbf{y}}^{2}\right) .
$$

It is worth noting that, in general, the elements of $\sigma_{\mathbf{y}}^{2}$ can be different from each other, so that the diagonal elements of $\mathbf{Q}_{k}$ are not all equal. In this paper, the parameter $N$ has been set equal to 30. Appendix A reports a sensitivity analysis of the DKF-SE with respect to $N$ and justifies this choice. Section IV-C includes the DKF process model validation when the process noise covariance matrix $\mathbf{Q}_{k}$ is assessed according to (27)-(29).

\section{DKF-SE Versus LWLS-SE}

In this section, we compare theoretically the accuracy of DKF-SE versus LWLS-SE. The DKF algorithm makes use of all the available measurements, past and present, whereas the LWLS algorithm uses only measurements of the current time step. The former should perform better, provided that the process model hypotheses that underlie the DKF are correct. The following theorem makes this more precise; it states that the estimation error with the DKF algorithm is always less than the estimation error with the LWLS algorithm, the difference being given equal to the mean square difference between the two methods.

Theorem 1: Assume that the true (unobserved) state $\mathbf{x}_{k}$ satisfies the process model in (15). Assume that the system parameters defined in Section III-B are known. Let $\widehat{\mathbf{x}}_{k \text {,LWLS }}$ and $\widehat{\mathbf{x}}_{k, \mathrm{DKF}}$ be the state estimates obtained at time step $k$ with the LWLS and DKF algorithms, respectively. Then

$$
\begin{aligned}
\mathbb{E}\left(\left\|\mathbf{x}_{k}-\widehat{\mathbf{x}}_{k, \mathrm{LWLS}}\right\|^{2}\right)= & \mathbb{E}\left(\left\|\mathbf{x}_{k}-\widehat{\mathbf{x}}_{k, \mathrm{DKF}}\right\|^{2}\right) \\
& +\mathbb{E}\left(\left\|\widehat{\mathbf{x}}_{k, \mathrm{LWLS}}-\widehat{\mathbf{x}}_{k, \mathrm{DKF}}\right\|^{2}\right) .
\end{aligned}
$$

Proof: First, let us observe that by standard KF theory (see [52, p. 148]), the estimation of the nonobservable state is equal to its conditional expectation, given the sequence of observations, that is

$$
\widehat{\mathbf{x}}_{k, \mathrm{DKF}}=\mathbb{E}\left(\mathbf{x}_{k} \mid \mathcal{F}_{k}\right)
$$

where $\mathcal{F}_{k}$ is the $\sigma$ field generated by all measurements up to and including time step $k$.

Second, consider the Hilbert space of random vectors (with values in $\mathbb{R}^{S}$ ) equipped with the inner product $\langle\mathbf{X}, \mathbf{Y}\rangle_{H} \triangleq \mathbb{E}\left(\sum_{r=1}^{S} \mathbf{X}(r) \mathbf{Y}(r)\right)$. We now show that the random vector $\mathbf{x}_{k}-\widehat{\mathbf{x}}_{k, \mathrm{DKF}}$ is orthogonal, in the sense of this Hilbert space, to all random vectors $\mathbf{Y}$ that are $\mathcal{F}_{k}$-measurable (i.e., that are a function of the observations up to time $k$-in this context, the initial conditions of the estimation algorithms are assumed to be known and nonrandom). Note that what we want to show is

$$
\mathbb{E}\left(\left\langle\mathbf{Y}, \mathbf{x}_{k}\right\rangle\right)=\mathbb{E}\left(\left\langle\mathbf{Y}, \widehat{\mathbf{x}}_{k, \mathrm{DKF}}\right\rangle\right)
$$

with $\left\langle\mathbf{Y}, \mathbf{x}_{k}\right\rangle=\sum_{r} \mathbf{Y}(r) \mathbf{x}_{k}(r)$.
In order to prove (32), observe that for any (real-valued) random variable $Y$ that is measurable with respect to $\mathcal{F}_{k}$, we have $\mathbb{E}\left(Y \mid \mathcal{F}_{k}\right)=Y$ and further [52, Eq. (1.7.15)]

$$
\mathbb{E}\left(Y \mathbf{x}_{k}(r) \mid \mathcal{F}_{k}\right)=Y \mathbb{E}\left(\mathbf{x}_{k}(r) \mid \mathcal{F}_{k}\right)=Y \widehat{\mathbf{x}}_{k, \mathrm{DKF}}(r) .
$$

Note that such a $Y$ can be any nonlinear real-valued function of $\left(\mathbf{z}_{1}, \ldots, \mathbf{z}_{k}\right)$. Take expectations on both sides and use the fact that the expectation of the conditional expectation is the same as the original expectation [52, Eq. (1.7.14)] and obtain

$$
\mathbb{E}\left(Y \mathbf{x}_{k}(r)\right)=\mathbb{E}\left(Y \widehat{\mathbf{x}}_{k, \operatorname{DKF}}(r)\right) .
$$

Consider now any $\mathcal{F}_{k}$-measurable random vector $\mathbf{Y}$, apply (33) to $Y=\mathbf{Y}(r)$ for all coordinates $r$, and sum over $r$, then it becomes

$$
\mathbb{E}\left(\sum_{r} \mathbf{Y}(r) \mathbf{x}_{k}(r)\right)=\mathbb{E}\left(\sum_{r} \mathbf{Y}(r) \widehat{\mathbf{x}}_{k, \mathrm{DKF}}(r)\right)
$$

which shows the above equation as required.

Now observe that both $\widehat{\mathbf{x}}_{k, \text { LWLS }}$ and $\widehat{\mathbf{x}}_{k, \text { DKF }}$ are $\mathcal{F}_{k}$-measurable because they are derived from the measurements. Therefore, we can apply the previous result to $\mathbf{Y}=\widehat{\mathbf{x}}_{k \text {,LWLS }}-\widehat{\mathbf{x}}_{k, \text { DKF. Equation (30) then follows }}$ from Pythagoras's equality.

The theorem applies as long as the process model in (15) holds. This is why it is important to verify the adequacy of the process model, as we do in Section IV-C.

\section{Performance Evaluation}

In this section, we evaluate the performance of the LWLS-SE and the DKF-SE described in Section III.

The use of synchrophasor measurements in real-time SE is, from the problem formulation perspective, independent of the peculiar nature of the analyzed grid. For this reason, we have decided to discuss the performance assessment of linear state estimators for the two cases of transmission and distribution grids. These systems have different characteristics in terms of topology, line parameters, and operation conditions, and the purpose of this section is to numerically support the conclusions that have been analytically given in Section III. This performance analysis is carried out using two case studies, namely, a distribution (IEEE 123-bus test feeder [30]) and a transmission (IEEE 39-bus test system [31]) network. The procedure adopted to perform the SE is as follows.

1) Each 20 milliseconds, a power flow is computed in order to determine the true state of the network.

2) The measurements forwarded to the $\mathrm{SE}$ are obtained by perturbing the true quantities inferred from the previous step with randomly generated Gaussian noise characterized by the cumulated standard deviation of the PMUs and their sensors. We assume to use PMUs of class-P. This choice is motivated by the fact that active distribution networks (ADNs) are characterized by larger distortion levels and dynamic behaviors compared with transmission networks. In addition, as they might be operated even islanded from main transmission networks, the application of PMUs to monitor electromechanical transients needs to be robust against 
TABLE I

Limits of Ratio ERRoR AND PHASE DisPlacement OF THE USED VOLTAGE SENSORS ACCORDING TO [53] AND [55]

\begin{tabular}{|c|c|c|}
\hline Class & Ratio error [\%] & Phase displacement [radians] \\
\hline 0.1 & 0.1 & $1.5 \cdot 10^{-3}$ \\
\hline 0.5 & 0.5 & $6 \cdot 10^{-3}$ \\
\hline 1 & 1 & $12 \cdot 10^{-3}$ \\
\hline
\end{tabular}

TABLE II

Limits of RATIO ERROR AND PHASE DisPLACEMENT OF THE USED CURRENT SENSORS ACCORDING TO [53] AND [54]

\begin{tabular}{|c|c|c|}
\hline Class & Ratio error [\%] & Phase displacement [radians] \\
\hline 0.1 & 0.1 & $1.5 \cdot 10^{-3}$ \\
\hline 0.5 & 0.5 & $9 \cdot 10^{-3}$ \\
\hline 1 & 1 & $18 \cdot 10^{-3}$ \\
\hline
\end{tabular}

TABLE III

Limits of MAGNitude AND PHASE ERRORS FOR THE USED PMUs ACCORDING TO [9]

\begin{tabular}{|c|c|c|}
\hline TVE (in \%) & Magnitude error [\%] & Phase error [radians] \\
\hline 0.14 & 0.1 & $10^{-3}$ \\
\hline
\end{tabular}

important misestimation of synchrophasor phases and frequencies [20]. For transmission network applications, this choice is also justified by the possibility to use these devices, coupled with associated state estimators, for protection purposes typically involving fault location functionalities. The voltage and current sensors used to perform the tests are assumed to be of 0.1-, 0.5-, and 1-class and the limits of ratio error and phase displacement imposed by [53]-[55] are shown in Tables I and II. The limits of magnitude and phase errors for the adopted PMUs are shown in Table III and correspond to a total vector error (TVE) equal to $0.14 \%$. This TVE value results from assuming PMU class-P devices characterized by typical maximum errors in magnitude and phase of $0.1 \%$ and $10^{-3}$ rads, respectively. The cumulated error of sensor and PMU is divided by three in order to get the standard deviations of the Gaussian noises. It is important to mention that the measurement noise covariance matrix $\mathbf{R}_{k}$ changes at every time step, since the measurement errors are here defined in polar coordinates, while the state is expressed in rectangular coordinates.

3) Then, based on these measurements, the system state is calculated using the considered state estimators.

\section{A. Distribution Network Case}

1) Network Description: The IEEE 123-bus distribution test feeder [30] is the first network adopted to evaluate the performance of the aforementioned state estimators. We assume that the network has a rated voltage equal to $15-\mathrm{kV}$ line-to-line RMS and the lines are unbalanced, corresponding to the \#602 line configuration of the IEEE
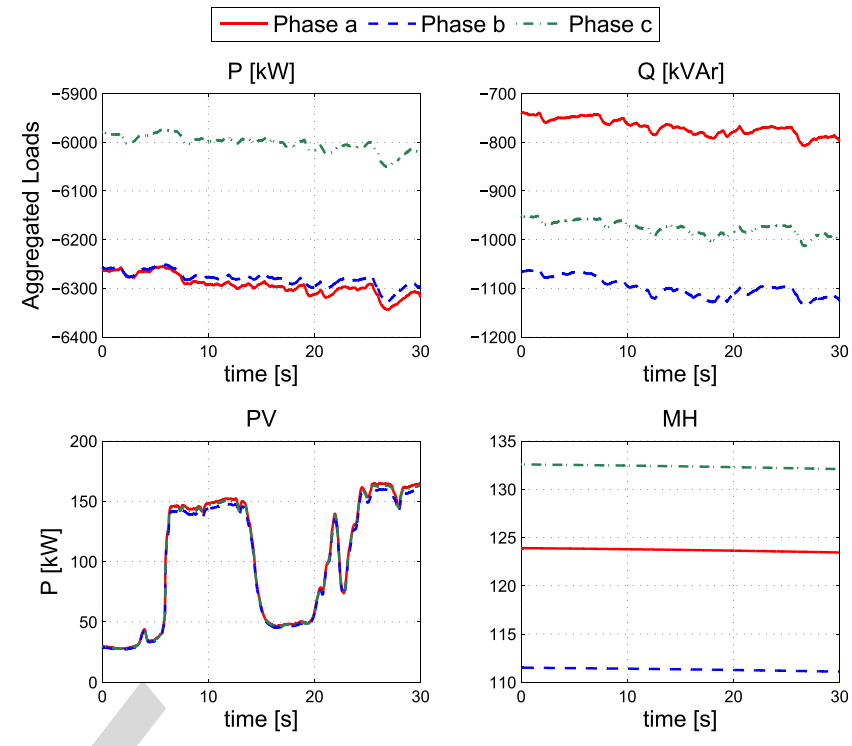

Fig. 1. Power profiles per phase used in the IEEE 123-bus test feeder. (a) Aggregated active and reactive power profiles of the loads. (b) Active power profiles of the two DERs (PV and MH).

distribution test feeders [30]. The loads/distributed energy resources (DERs) are also characterized by unbalanced power absorptions/injections, respectively. Bus \#150 of the configuration reported in [30] represents the connection to a subtransmission network with a short-circuit power $S_{\mathrm{sc}}=300$ MVA and a ratio between the real and imaginary parts of the short-circuit impedance $R_{\mathrm{sc}} / X_{\mathrm{sc}}=0.1$.

Fig. 1 shows the aggregated active and reactive power consumption of the loads and the active power that is injected by the DERs in the three phases over a time window of $30 \mathrm{~s}$. Two DERs are connected to the 123-bus network, one photovoltaic (PV) installation in Bus \#92 and a minihydro (MH) power plant in Bus \#112. It is assumed that the DERs inject only active power. Loads are connected to 85 network buses. It is important to mention that the loads and PV power profiles come from real measurements obtained from PMUs installed in the 20-kV distribution network of the Swiss Federal Institute of Technology of Lausanne [18]. The MH power profiles come from another real distribution network in Switzerland. All these measurements have been obtained with a frame rate of 50 frames-per-second. We would like to clarify why we used measurement data coming from real networks and not the ones included in [30]. Reference [30], which gives test distribution network characteristics, provides only static load powers, while we need to use varying power profiles with sub-second frame-rate since we target to assess the accuracy of real-time state estimators. In the case of static profiles, the DKF obviously provides better state estimates since the process model is perfectly known and it effectively filters the measurement noise. In presence of state variations, it is not trivial to show that its performance can be similar or better than the one of LWLS. Indeed, the adopted process model has to be integrated with a method that assesses the process noise covariance matrix during the simulation, in order to adapt to the state variations. 
TABLE IV

PMU LOCATIONS AND ZERO-INJECTION BUSES IN THE IEEE 123-BUS TEST FEEDER

\begin{tabular}{|c|c|c|}
\hline Case & Number & Location \\
\hline \multirow{4}{*}{ Zero-injection buses } & & 381314151821232526 \\
& & 27364044545761677278 \\
& & 149151250300450610 \\
\hline & & 2469111216202224 \\
& & 29323334353739414346 \\
& & 48495152565962646669 \\
& & 717375778083858688 \\
& & 109929698100102104 \\
& & 107112114150 \\
\hline
\end{tabular}

For the sake of comparison, all the state estimators use measurements streamed at 50 frames-per-second [9], [10] and the same measurement location. The assumed PMU locations and the zero-injection buses are given in Table IV. The measurement placement is chosen in order to fulfill the system observability (H matrix is of full rank; see Section III) and achieve a good SE accuracy with a minimum number of PMUs. Indeed, the number and location of PMUs do not affect the conclusions of this paper (see Appendix B), and consequently, the optimal placement of PMUs has not been treated in this paper.

2) SE Accuracy and Timing Assessment: Fig. 2 shows the time profiles of the maximum errors of the estimated state versus the true one for the LWLS-SE and DKF-SE, for a time window of $30 \mathrm{~s}$ and a resolution of $20 \mathrm{~ms}$. The errors refer to both voltage magnitude $V$ and phase $\delta$. The magnitude error is expressed in percentage of the amplitude of the true quantity and the phase error is expressed in radians. At time step $k$, the maximum estimation error is calculated considering the estimation errors in all the buses and the three phases as

$$
\begin{aligned}
& \operatorname{err} V_{\text {MAX }_{k}}=\max \left[\operatorname{err} V_{1, k}^{a}, \operatorname{err} V_{1, k}^{b}, \operatorname{err} V_{1, k}^{c}, \ldots,\right. \\
& \left.\operatorname{err} V_{n, k}^{a}, \operatorname{err} V_{n, k}^{b}, \operatorname{err} V_{n, k}^{c}\right] \\
& \operatorname{err} \delta_{\mathrm{MAX}_{k}}=\max \left[\operatorname{err} \delta_{1, k}^{a}, \operatorname{err} \delta_{1, k}^{b}, \operatorname{err} \delta_{1, k}^{c}, \ldots,\right. \\
& \left.\operatorname{err} \delta_{n, k}^{a}, \operatorname{err} \delta_{n, k}^{b}, \operatorname{err} \delta_{n, k}^{c}\right] .
\end{aligned}
$$

Simulations have been carried out for the three different accuracy classes of voltage and current sensors given in Tables I and II. Both the magnitude and the phase maximum errors of the LWLS-SE are larger than the ones of the DKF-SE along the overall simulation time and for all the considered sensor accuracy classes. In order to further clarify this aspect, Fig. 3 shows the profiles of the ratio between the LWLS and the DKF maximum voltage magnitude and phase errors, calculated using (35), for a time window of $30 \mathrm{~s}$ and a resolution of $20 \mathrm{~ms}$. The used measurement sensors are of 0.1-class. As it can be observed, the ratio between the LWLS and the DKF maximum errors is most of the time between 4 and 6 , with upper values of 10 . Similar results hold for the 0.5 - and 1-class sensors. These results quantify the

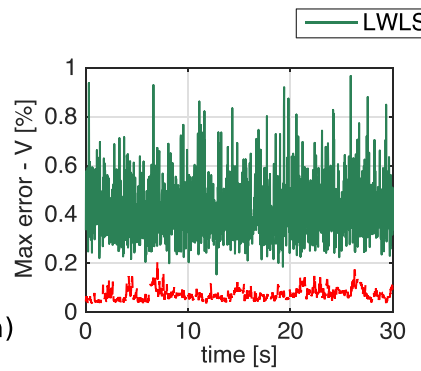

WLS - - DKF
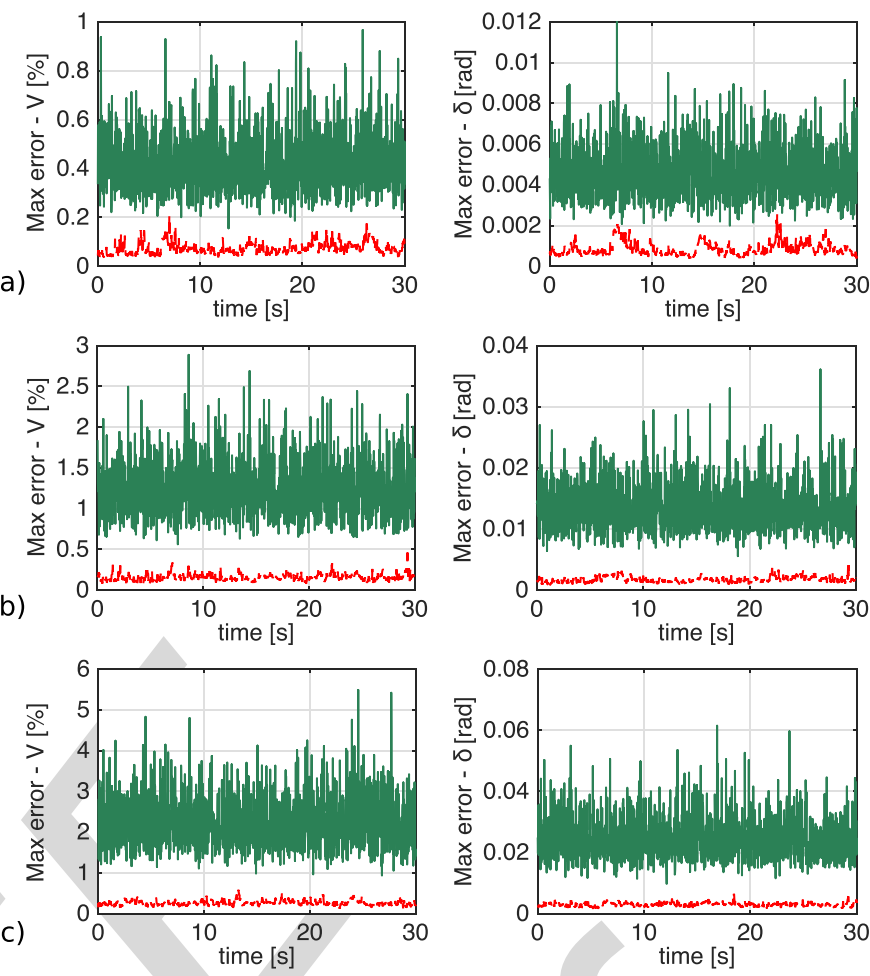

Fig. 2. Time profiles of the maximum estimation errors, calculated using (35), for the LWLS-SE and DKF-SE, in the case of the IEEE 123-bus test feeder. The magnitude is in percentage of the amplitude of the true quantity, while the phase is in radians. Three sensor accuracy classes have been considered. (a) 0.1-class. (b) 0.5-class. (c) 1-class.
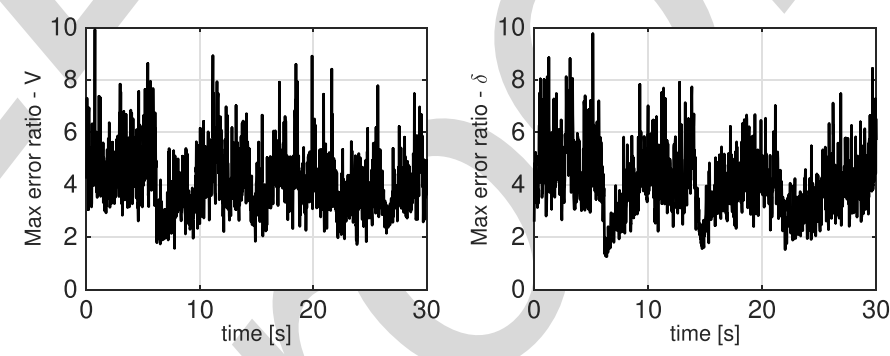

Fig. 3. Time profiles of the ratio between the LWLS and the DKF maximum voltage magnitude and phase errors, calculated using (35), in the case of the IEEE 123-bus test feeder with 0.1-class measurement sensors.

non-negligible better peformance of the DKF-SE over the LWLS-SE that can indeed reach one order of magnitude.

To provide a better understanding of the behavior of the two state estimators, Fig. 4 shows the time evolution of the true and estimated voltage at Bus \#92, phase $a$. This bus is connected to the PV plant and, as a consequence, it is the one experiencing, in general, the largest changes in the state. The LWLS estimates are mainly affected by the measurement noise, while the DKF is able to filter it out effectively. In addition, it is possible to observe that the DKF has a short delay in tracking fast state variations, and even in this case, its estimation errors remain lower than the ones of the LWLS. This capability of the DKF is due to the way the process model covariance matrix is updated via (27)-(29). In this respect, 

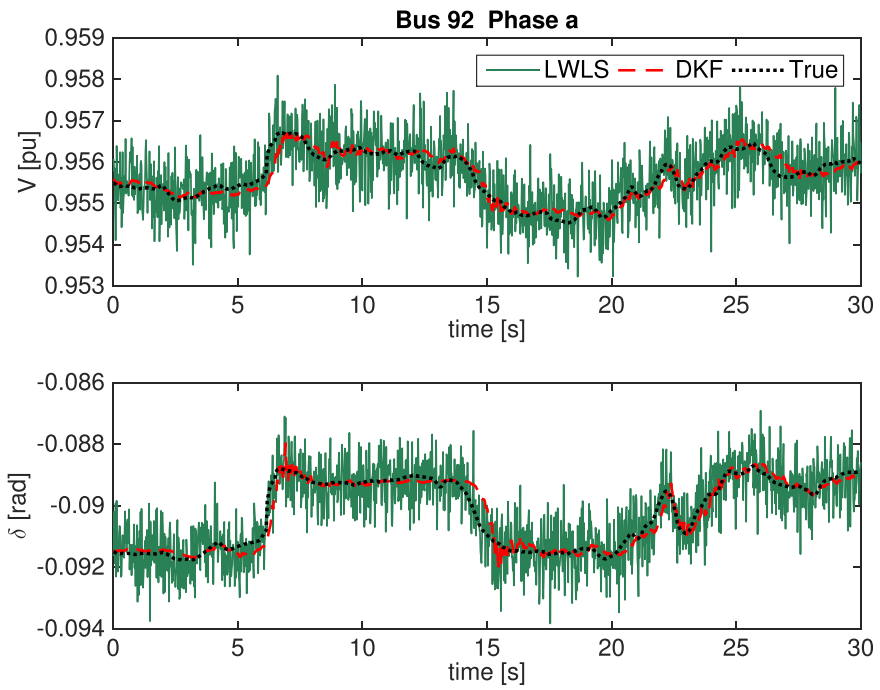

Fig. 4. Time evolution of the voltage magnitude and phase at Bus \#92, phase $a$, with a time window of $30 \mathrm{~s}$, in the case of the IEEE 123-bus test feeder with 0.1-class sensors: true state (dotted black lines), LWLS estimate (continuous green lines), and DKF estimate (dashed red lines).
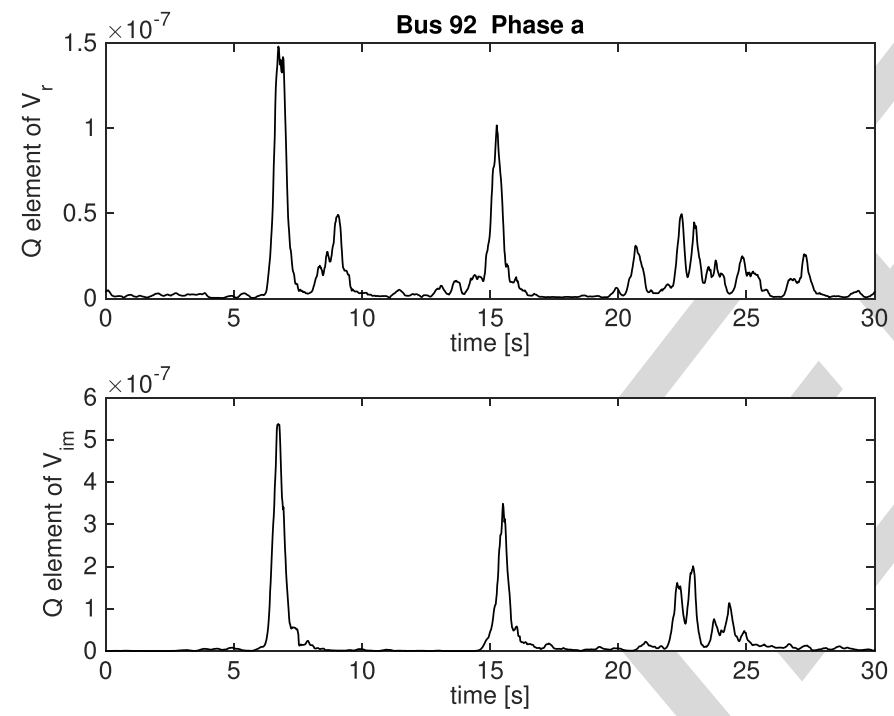

Fig. 5. Time evolution of the elements of the process noise covariance matrix corresponding to the real and imaginary parts of the voltage at Bus \#92, phase $a$, with a time window of $30 \mathrm{~s}$, in the case of the IEEE 123-bus test feeder with 0.1-class sensors.

Fig. 5 shows the time evolution of the elements of the process noise covariance matrix for the real and imaginary parts of the voltage at Bus \#92, phase $a$. It is possible to observe the inflation of these elements of the process noise covariance matrix in correspondence with fast variations of the state at $t=7,15$, and $23 \mathrm{~s}$.

It is also worth observing that the maximum errors of LWLS are significantly larger than the ones of the measurements (see Tables I and III). This is due to the fact that the maximum error takes account of all the network buses: the estimation errors at the buses without PMUs can be up to three times larger than the ones at the buses equipped with a PMU.

The numerical evaluation shown in Fig. 2 confirms the formal proof of the better accuracy of the DKF-SE over the
TABLE V

COMPUTATION TIMES OF THE CONSIDERED STATE Estimators FOR THREE DifFERENT Distribution TEST FEEDERS

\begin{tabular}{|c|c|c|}
\hline \multicolumn{3}{|c|}{ Mean \pm standard deviation [ms $]$} \\
\hline Network & LWLS-SE & DKF-SE \\
\hline IEEE 13-bus & $0.36 \pm 0.070$ & $0.65 \pm 0.088$ \\
\hline IEEE 34-bus & $1.4 \pm 0.22$ & $2.8 \pm 0.31$ \\
\hline IEEE 123-bus & $42 \pm 4.3$ & $73 \pm 4.6$ \\
\hline
\end{tabular}

LWLS-SE presented in Section III-D. In addition, the amplitudes of the maximum voltage magnitude and phase errors prove the possibility to track with high quality and fidelity the system state of ADNs using the DKF-SE where the $\mathbf{Q}$ matrix is properly assessed.

Concerning the SE timing performance, Table V shows the computation times (in milliseconds) of the considered state estimators in terms of mean and standard deviation values, for the IEEE 13, 34 and 123-bus distribution test feeders [30]. These values have been inferred by implementing the SE algorithms described in Sections III-A and III-B in an Apple MacBook Pro with a 2.6-GHz CPU, 8-GB RAM, and MATLAB 2014b. The IEEE 13-bus test feeder is equipped with seven PMUs, whereas the IEEE 34-bus test feeder is equipped with 12 PMUs and has 15 zero-injection buses. A simulation of $30 \mathrm{~s}$ has been considered (i.e., 1500 estimates). As it can be observed, the computation time increases when the network size becomes larger. The LWLS-SE is faster than the DKF-SE for all the tested distribution feeders. However, even for large systems, such as the 123-bus distribution test feeder, the computational cost remains modest: its mean is $78 \mathrm{~ms}$ for the largest network topology and below $2.8 \mathrm{~ms}$ for the other ones. It is important to note that even for the case of feeders with an extremely large number of buses, the DKF-SE is characterized by computation times below $100 \mathrm{~ms}$, which are compatible with real-time applications. In case SE refresh rate of 50 frames-per-second is needed, the state estimators can be suitably pipelined (see Section IV-D).

\section{B. Transmission Network Case}

1) Network Description: The IEEE 39-bus test system [31] represents the transmission network case used to evaluate the performance of the considered state estimators. The network is balanced, and therefore, only the direct sequence has been considered. Bus \#31 of the configuration reported in [31] is the connection point of the system to an external grid characterized by a short-circuit power $S_{\mathrm{sc}}=50 \mathrm{GVA}$ and a ratio between the real and imaginary parts of the short circuit impedance $R_{\mathrm{sc}} / X_{\mathrm{sc}}=0$, which is a standard assumption for transmission power systems. The assumed PMU locations and the zero-injection buses are given in Table VI. The absorbed/injected powers are the ones reported in [31]. As in Section IV-A, we ensured that matrix $\mathbf{H}$ has full rank.

2) SE Accuracy and Timing Assessment: Fig. 6 shows the time profiles of the maximum errors of the estimated state versus the true one for the LWLS-SE and DKF-SE. As in the 
TABLE VI

PMU LOCATIONS AND ZERO-INJECTION BUSES IN THE IEEE 39-BUS TEST SYSTEM

\begin{tabular}{|c|c|c|}
\hline Case & Number & Location \\
\hline Zero-injection buses & 12 & $\begin{array}{r}125691011 \\
1314171922\end{array}$ \\
\hline PMUs & 19 & $\begin{array}{r}471215182124272830 \\
313233343536373839\end{array}$ \\
\hline
\end{tabular}
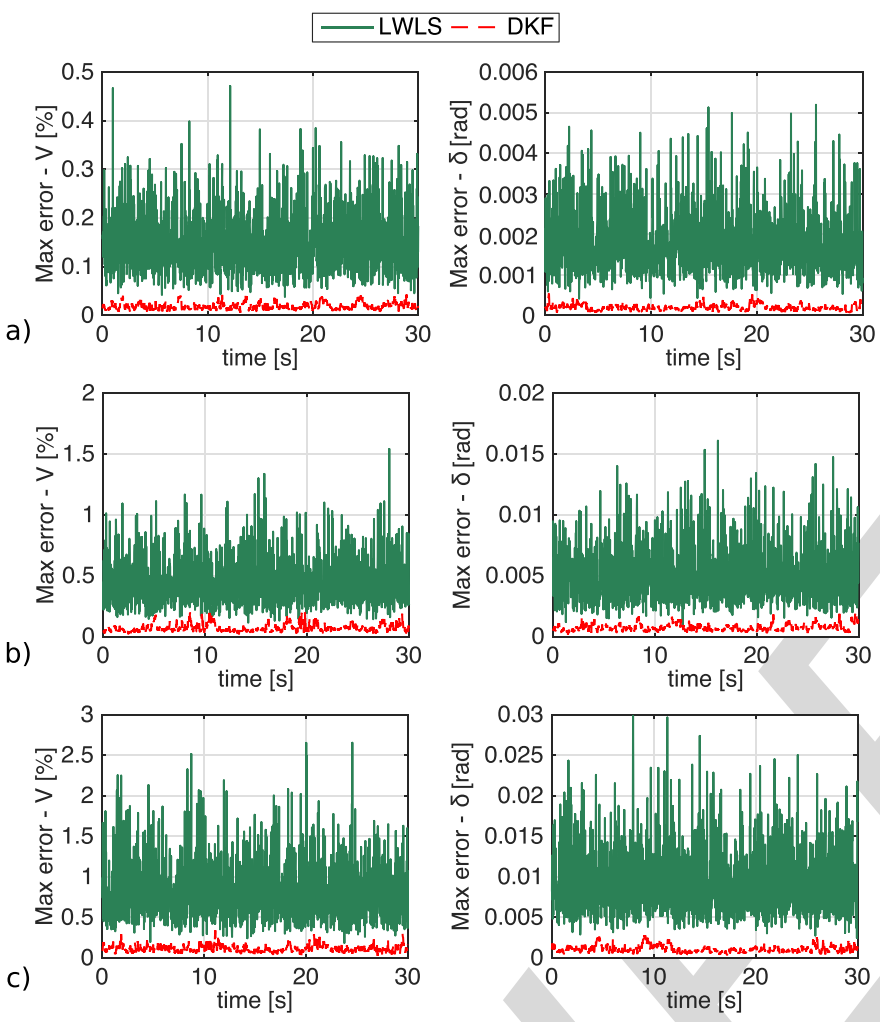

Fig. 6. Time profiles of the maximum estimation errors, calculated using (35), for the LWLS-SE and DKF-SE, in the case of the IEEE 39-bus test system. The magnitude is in percentage of the amplitude of the true quantity, while the phase is in radians. Three sensor accuracy classes have been considered. (a) 0.1-class. (b) 0.5-class. (c) 1-class.

distribution network case, the maximum estimation errors are calculated using (35) and we consider three different accuracy classes of voltage and current sensors given in Tables I and II. We can observe that also for the transmission system case, both the magnitude and the phase maximum errors of the LWLS-SE are larger than the ones of the DKF-SE along the overall simulation time and for all the considered sensor accuracy classes. In addition, the ratio between the LWLS and the DKF maximum errors is similar to the one of the distribution network, reported in Section IV-A.

The results shown in Figs. 2 and 6 for the distribution feeder and the transmission system, respectively, indicate the better accuracy of the DKF-SE in both cases.

Concerning the SE timing performance, Table VII shows the computation times (in milliseconds) of the considered state estimators in terms of mean and standard deviation values for the IEEE 39-bus test system. The LWLS-SE computes the
TABLE VII

COMPUTATION TIMES OF THE CONSIDERED STATE Estimators FOR THE IEEE 39-BUS TEST SYSTEM

\begin{tabular}{|c|c|c|}
\hline \multicolumn{3}{|c|}{ Mean \pm standard deviation [ms] } \\
\hline Network & LWLS-SE & DKF-SE \\
\hline IEEE 39-bus & $0.29 \pm 0.061$ & $0.39 \pm 0.080$ \\
\hline
\end{tabular}

system state faster than the DKF-SE and has the smallest deviation from the mean value. The computation times of the IEEE 39-bus transmission test system are close to the ones of the IEEE-13 bus distribution test feeder since they are characterized by the same number of state variables and similar number of processed measurements.

\section{DKF Process Model Validation}

In Section III-B, we described the DKF algorithm. The validity of this method depends on how accurate the underlying system model is. More precisely, since the DKF equations use only second-order properties, it is sufficient to verify that the covariance properties of the model do hold. We thus need to verify whether the residuals $\widehat{\mathbf{x}}_{k+1}-\widehat{\mathbf{x}}_{k}$ are noncorrelated. We start with the analysis for the distribution network, described in Section IV-A. For brevity, we analyze Bus \#61 since it is the bus that shows the worst performance in terms of estimation error. The results are shown with respect to the real and imaginary parts of the voltage. However, the same conclusions are valid for all the other buses.

It is worth mentioning that the residuals are normalized by the corresponding values of the process noise covariance matrix $\mathbf{Q}_{k}$ since it is continuously assessed with the method described in Section III-C.

We first plot the normalized residuals themselves in Fig. 7(a) (real part of the voltage for the three phases) to check whether the stationarity assumption is satisfied, which is the case. A more formal verification is to plot and analyze the sample autocorrelation functions (ACFs) as in Fig. 7(b). We do it for the first $\sim \sqrt{n}$ lags, where $n=1500$ is the number of samples. If the residuals are noncorrelated, the ACFs should be within the noise margins $\pm 1.96 / \sqrt{n}$ with $95 \%$ of probability [56]. We observe from Fig. 7(b) that the above-mentioned condition is fulfilled and, consequently, that our model is accurate. Fig. 8(a) and (b) shows the same results for Bus \#15 of the IEEE 39-bus transmission test system (real part of the voltage balanced network). Similar results are obtained for the rest of the transmission network buses. The ACF is, as a matter of fact, a statistically distributed quantity. The fact that, in few cases, the ACFs are slightly beyond the noise margins does not violate the validity of the result and the numerical proof of the statistical correctness of the process model. For brevity, we do not show model validation plots for the imaginary part of the voltage. However, we do confirm that the same conclusions hold as the residuals are stationary and the corresponding ACFs reveal no correlation.

Furthermore, we also verify whether the quantitative conclusion of Theorem \#1 of Section III-D, namely (30), numerically holds. In Fig. 9 we, respectively, show the 

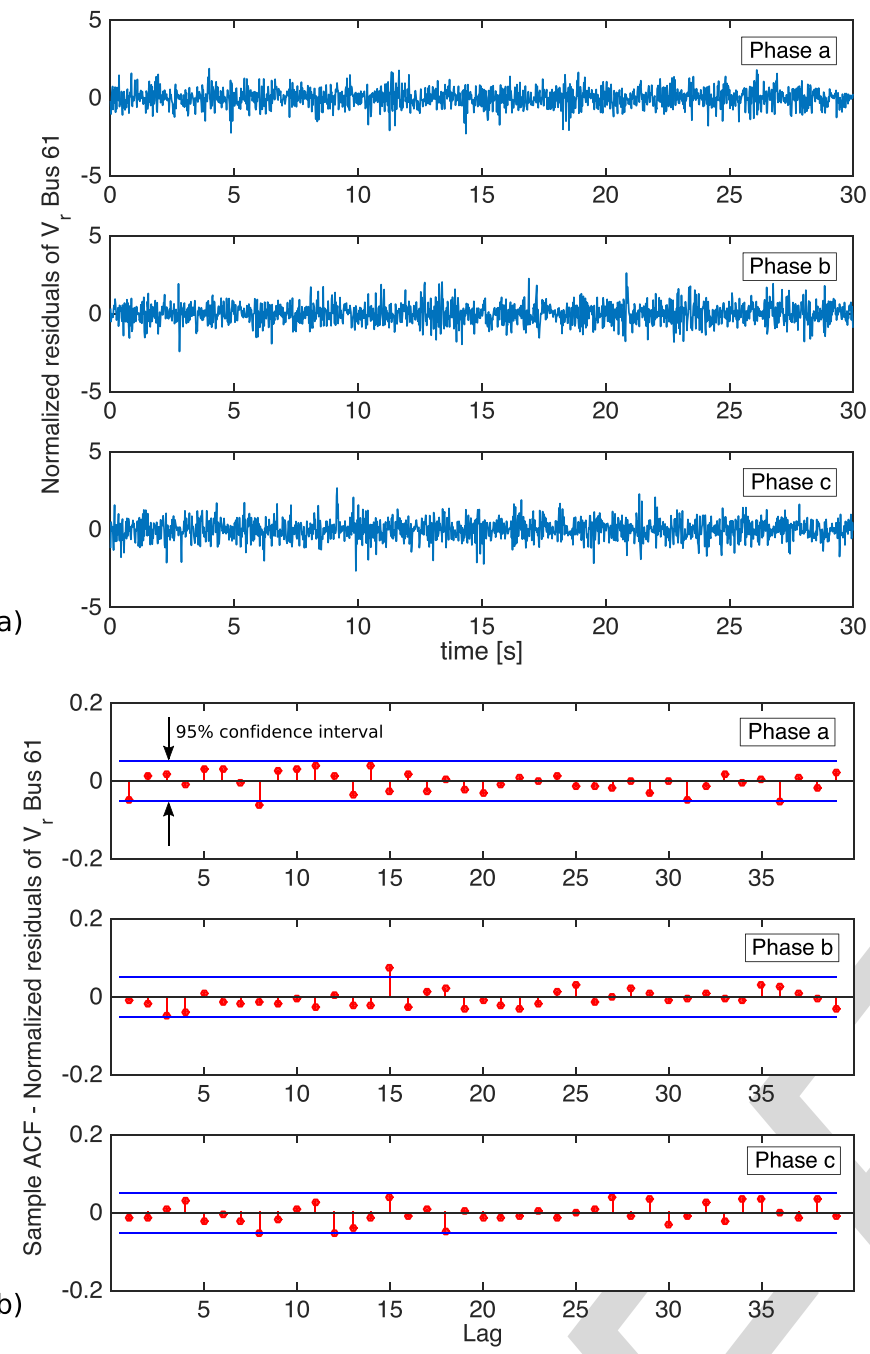

Fig. 7. DKF process model validation with respect to the real part of the voltage at Bus \#61 of the IEEE 123-bus distribution test feeder, which exhibits the worst performance in terms of estimation error. (a) Residuals are stationary and sample ACFs (b) are within the noise level bars, which confirm that the correlation is negligible (similar results obtained for the imaginary part and for the rest of the network buses).

left-hand side (LHS) and right-hand side (RHS) of (30) for the case of the distribution network, where it appears that both are very close to each other. For the sake of space, since the network is 3-ph and consists of 123 buses, we show the first ten buses. Similarly to Fig. 9, Fig. 10 shows the LHS and RHS of (30) for the case of the transmission network. As it can be observed, also in this case, the RHS and LHS of (30) are close to each other. At this point, it is important to note that the equality in Theorem \#1 is in expectation. In Figs. 9 and 10, we estimate the expectations by empirical averages, and therefore, a small discrepancy is expected. Figs. 9 and 10 also show the contributions of the two terms of the RHS of (30). It can be observed that the contribution of the second one is predominant with respect to the first one, proving that the DKF is applied correctly.

\section{SE Pipelining and Latency}

First, we would like to clarify the difference between refresh rate and latency. We aim to have SE performed at a refresh

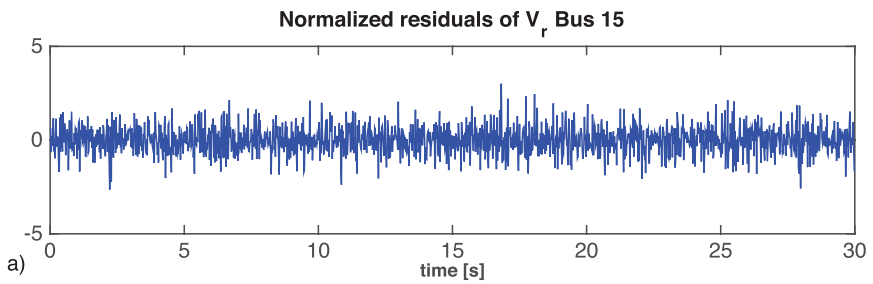

Sample ACF - Normalized residuals of $V_{r}$ Bus 15

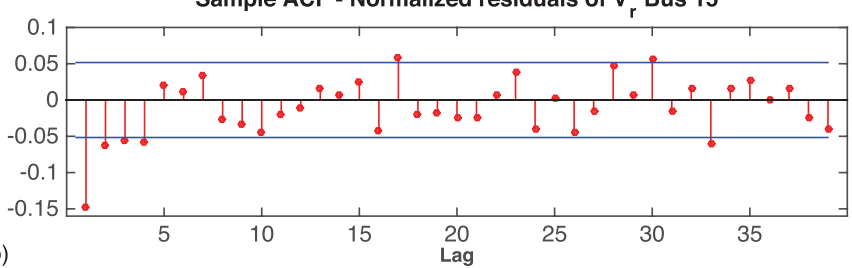

Fig. 8. DKF process model validation with respect to the real part of the voltage at Bus \#15 phase $a$ of the IEEE 39-bus transmission test system. (a) Residuals are stationary and sample ACFs (b) are within the noise level bars, which confirm that the correlation is negligible (similar results obtained for the imaginary part and for the rest of the network buses).

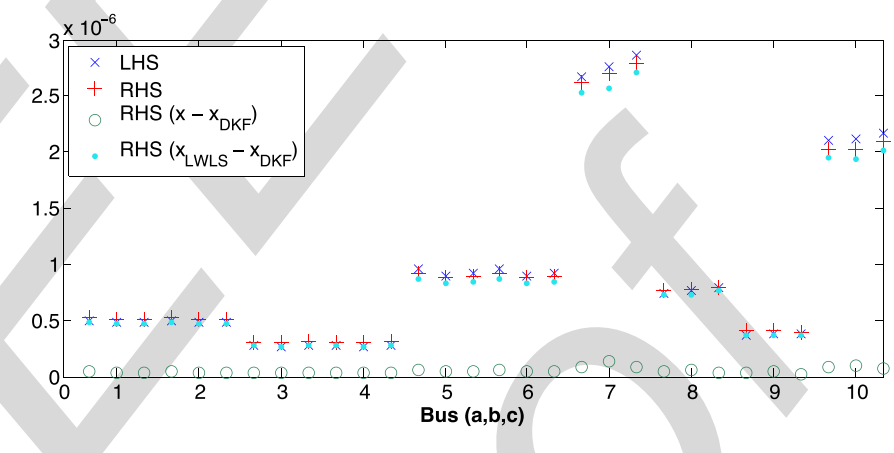

Fig. 9. Verification of (30) for the DKF-SE applied to the IEEE 123-bus distribution test feeder: LHS versus RHS of the equation. The separate contribution of the two terms of the RHS side is also shown.

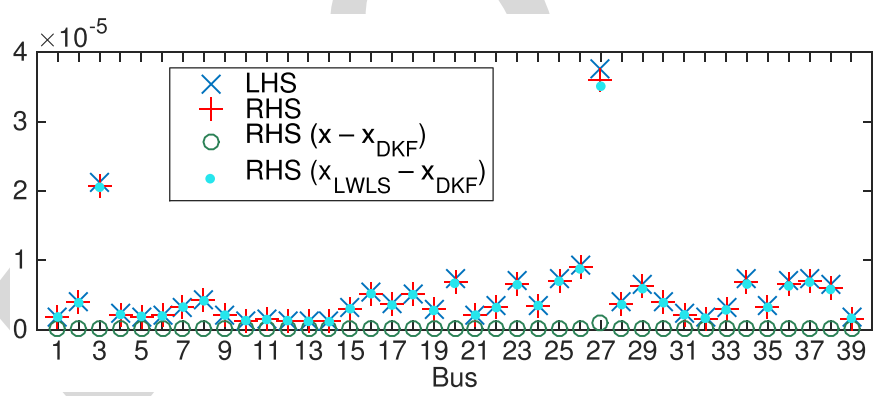

Fig. 10. Verification of (30) for the DKF-SE applied to the IEEE 39-bus transmission test system: LHS versus RHS of the equation. The separate contribution of the two terms of the RHS side is also shown.

rate of $20 \mathrm{~ms}$, but the total latency of the process in a real implementation might be higher as explained in what follows.

In particular, the latency is defined as the time starting from the time tag of a set of measurements that is forwarded to the $\mathrm{SE}$, up to the moment that the state is computed. Indeed, the total latency is the sum of the individual latencies of every component of the SE chain, i.e., measurement devices, telecom infrastructure, phasor data concentrator, and SE. A detailed 


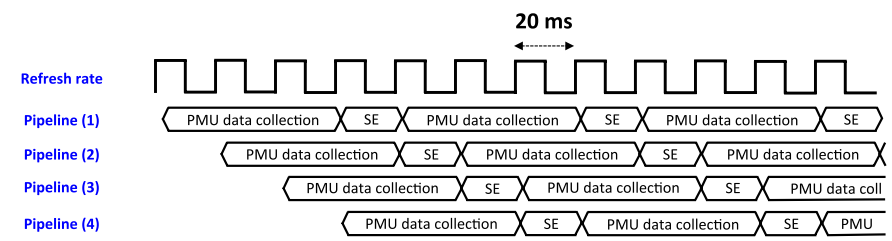

Fig. 11. Pipelining of the SE computation.

description of the latency assessment for an SE process of a real distribution feeder is given in [18] and [33].

In this respect, in case the total time to compute the state (i.e., the time for the PMU data collection as well as the SE, shown in Fig. 11) is above $20 \mathrm{~ms}$, but we want to keep a refresh rate of $20 \mathrm{~ms}$, the usual practice is to pipeline the SE computation. Several SEs are performed in parallel as shown in Fig. 11. The number of pipelines is calculated as

$$
N_{p l}=\operatorname{Int}\left(\frac{\text { PMU data collection time }+ \text { SE time }}{\text { Refresh rate }}\right) .
$$

In order to make it more clear, in the case that is shown in Fig. 11, if the PMU data collection takes three frames, namely, $60 \mathrm{~ms}$, these requirements can be satisfied by adopting a four-stage pipeline architecture. Each pipeline is typically assigned to a CPU core.

\section{CONCLUSIONS}

We have compared the performance of two different families of LSE processes, based on WLS (LWLS-SE) and KF (DKF-SE), that use synchrophasor measurements. The theory predicts that DKF-SE provides a better accuracy as long as its process model is correct. In the context of power systems, the process model that is classically used is given in (15). We have statistically validated its adequacy on the IEEE 123-bus distribution test feeder and the IEEE 39-bus transmission test system.

Numerical evaluations on these test systems confirm that the accuracy of DKF-SE is significantly better than the one of LWLS-SE. On the other hand, the computational cost of DKF-SE is larger than that of LWLS-SE. However, even in very large systems such as the 123-bus feeder, it remains modest: its mean is $73 \mathrm{~ms}$ for the largest network using a nonoptimized standard computer and below $2.8 \mathrm{~ms}$ for the other considered networks.

\section{APPENDIX A}

This Appendix provides further elements on the numerical validation of the method for the $\mathbf{Q}$ matrix assessment adopted in this paper. The DKF estimation results achieved with the adopted $\mathbf{Q}$ matrix assessment method (called DKF- $Q$ assessed) are compared with the ones obtained with a trial-and-error method (called $D K F-Q$ sampling) that uses a matrix $\mathbf{Q}$ that: 1 ) is kept constant along the simulation and 2) is diagonal with all the terms equal to a number $\widetilde{Q}$.

We here make reference to the case of the IEEE 123-bus distribution test feeder since distribution systems exhibit challenging operating conditions that are far from the quasi-steady state that characterizes transmission networks. Concerning this system (in the same operating conditions described
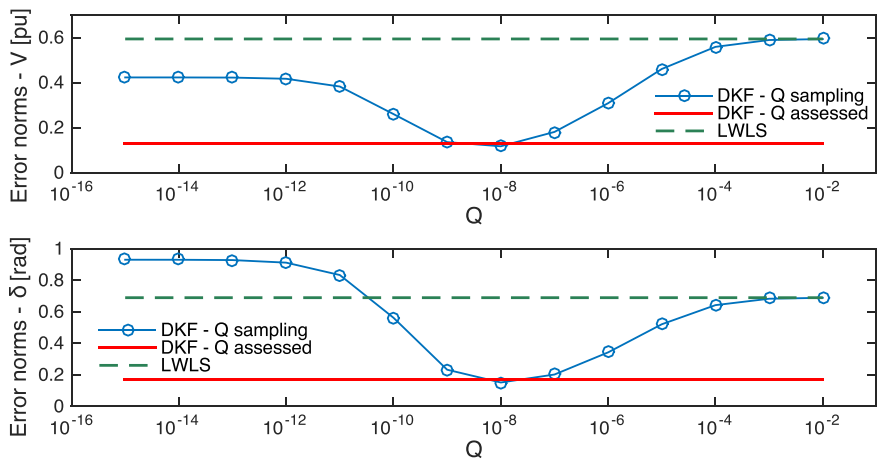

Fig. 12. Norms of the errors of the voltage magnitude and phase as a function of $\widetilde{Q}$ : comparison among the $D K F-Q$ sampling state estimators (blue curves), the $D K F-Q$ assessed (red lines), and the LWLS (green dashed lines).
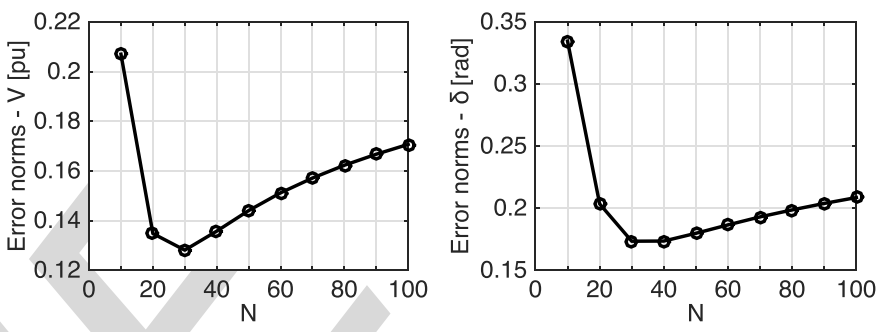

Fig. 13. Norms of the DKF estimation errors of the voltage magnitude and phase as a function of the parameter $N$.

in Section IV), Fig. 12 shows the curve of the norms of the estimation errors of the $D K F-Q$ sampling state estimators as a function of $\widetilde{Q}$ (blue lines with circles). The estimation errors at all the buses in all the three phases and for all the time steps are considered. The norm of the errors of the $D K F-Q$ assessed state estimator with the parameter $N$ set to 30 is represented by a red straight line since it does not depend on $\widetilde{Q}$ (note that the latter is a parameter related only to the $D K F-Q$ sampling state estimators). For the sake of completeness, Fig. 12 also includes the LWLS norm (green dashed lines). We can conclude that the adopted method for the on-the-fly assessment of $\mathbf{Q}$ is able to reach an estimation accuracy comparable with an a-posteriori trial-and-error method that uses constant values of $\mathbf{Q}$.

In addition, Fig. 13 shows the influence of the parameter $N$ on the estimation accuracy of the DKF-SE expressed in terms of norms of the errors of the voltage magnitude and phase as before (also in this case, the same conditions adopted to infer the results of Section IV have been used). The two graphs exhibit a minimum in correspondence with $N=30$ for both the voltage magnitude and phase errors. Indeed, a little value of $N$ (e.g., $N=10$ ) leads to an underestimate of $\mathbf{Q}$, and as a consequence, the DKF is incapable of following fast system state variations. On the contrary, if $N$ is too big (e.g., $N=100$ ), the $\mathbf{Q}$ is overestimated, and therefore, the estimates are significantly affected by the measurement noise. It has been observed that $N=30$ leads to good results in quasi-steady state conditions and also to a quick response by inflating $\mathbf{Q}$ when sudden events occur in the system, such as a load inrush or a fast increase/drop of the power injected by a PV plant (see Fig. 1). We would like to point out that we have used experimentally inferred time series of nodal absorptions/injections obtained from a PMU-based monitoring system installed at the Swiss Federal Institute of Technology 
TABLE VIII

DESCRIPTION OF THE TESTED PMU CONFiguRATIONS

\begin{tabular}{|c|c|c|}
\hline \multicolumn{3}{|c|}{ IEEE 123-bus distribution test feeder } \\
\hline Case & Buses with PMUs & Number of measurements \\
\hline Conf. 1 & $\begin{array}{rl}2446911 & 121620222429323334353739414346 \\
4849515256596264666971737577808385868890 \\
929698100102104107111112114 & 150\end{array}$ & 822 \\
\hline Conf. 2 & $\begin{array}{r}124691112162022242830323334353739414346 \\
4849515253565960626466697173757779808385 \\
86889092939698100102104107109111 \quad 112114150\end{array}$ & 906 \\
\hline Conf. 3 & $\begin{array}{l}124591012171922242829313334353738414345 \\
4849505253555860636566697173757779808385 \\
86889092939698100102104107109111112114150\end{array}$ & 906 \\
\hline Conf. 4 & $\begin{array}{r}124691011121617202224283032333435373941424346 \\
48495051525356596062646566686971737577798082 \\
838586889092939698100102104106107109111112114 \quad 150\end{array}$ & 1002 \\
\hline
\end{tabular}
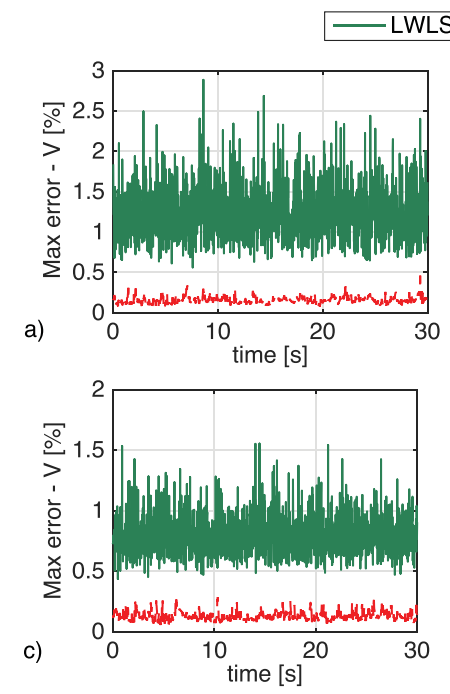
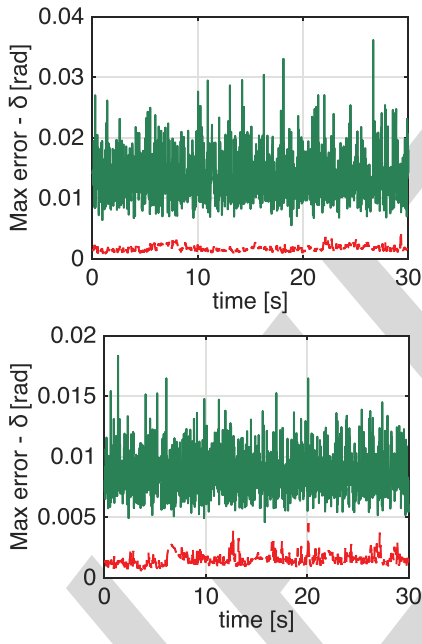
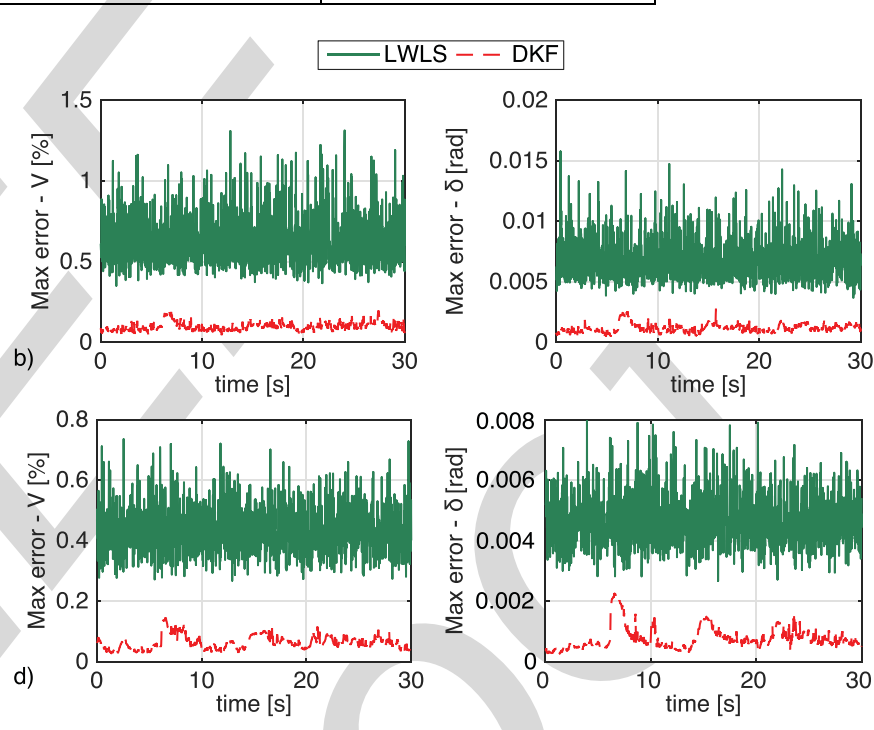

Fig. 14. Profiles of the maximum estimation errors, calculated using (35), for the LWLS-SE and DKF-SE, in the case of the IEEE 123-bus test feeder with 0.5 -class sensors. The magnitude is in percentage of the amplitude of the true quantity, while the phase is in radians. Four PMU configurations have been considered. (a) Configuration 1-51 PMUs. (b) Configuration 2-58 PMUs. (c) Configuration 3-58 PMUs (different PMU locations with respect to Configuration 2). (d) Configuration 4-66 PMUs.

of Lausanne [18]. Therefore, the assessment of the parameter $N$ is associated with realistic operating conditions. For grids that are mainly in quasi-steady state condition (it can be the case of the transmission grid used in this paper), a lower value of $N$ could be set, but the drawback is that in the case of events causing a quick change in the system state, the DKF will not be able to react properly.

\section{APPENDIX B}

The main purpose of this Appendix is to prove that the number and location of PMUs do not affect the conclusions of this paper. We work with a minimum number of PMUs in order to make the system fully observable, a condition that appears interesting for the network operators, since it minimizes the number of PMU installations. In our setting, we assume that each PMU provides 12 measurements to the LWLS and DKF: real and imaginary parts of the voltage and injected current, for every phase. In addition, each zero-injection bus is equivalent to six virtual measurements consisting of null injected currents.

For the sake of space, we here make reference to the case of the IEEE 123-bus distribution test feeder since the same results can be obtained for the IEEE 39-bus test system. In this respect, we have simulated the network with different numbers and locations of PMUs in order to show that these parameters do not significantly influence the comparison among the considered state estimators. We have tested the following four PMU configurations.

1) Configuration 1: The one used for the simulations in Section IV (51 PMUs).

2) Configuration 2: 58 PMUs.

3) Configuration 3: It is obtained from Conf. 2 by modifying the location of 12 PMUs.

4) Configuration 4: 66 PMUs.

Table VIII shows the buses equipped with PMUs as well as the number of measurements for each PMU configuration. 
The number of zero-injection buses is equal to 35 for all the above-mentioned cases, as reported in Table IV. Fig. 14 shows the time profiles of the maximum estimation errors for each PMU configuration, which correspond to the graphs of Section IV-A. It is evident that the number and location of PMUs do not affect the conclusions drawn in this paper regarding the comparison of the two linear state estimators.

\section{ACKNOWLEDGMENT}

The authors would like to thank Prof. L. Peretto for the useful discussion concerning the measurement sensors and, in particular, the associated accuracy.

\section{REFERENCES}

[1] A. Abur and A. Gómez Expósito, Power System State EstimationTheory and Implementation. New York, NY, USA: Marcel Dekker, 2004.

[2] A. Monticelli, State Estimation in Electric Power Systems: A Generalized Approach. Norwell, MA, USA: Kluwer, 1999.

[3] C. N. Lu, J. H. Teng, and W.-H. E. Liu, "Distribution system state estimation," IEEE Trans. Power Syst., vol. 10, no. 1, pp. 229-240, Feb. 1995.

[4] D. A. Haughton and G. T. Heydt, "A linear state estimation formulation for smart distribution systems," IEEE Trans. Power Syst., vol. 28, no. 2, pp. 1187-1195, May 2013.

[5] M. E. Baran and A. W. Kelley, "A branch-current-based state estimation method for distribution systems," IEEE Trans. Power Syst., vol. 10, no. 1, pp. 483-491, Feb. 1995.

[6] Z. Huang, K. Schneider, and J. Nieplocha, "Feasibility studies of applying Kalman filter techniques to power system dynamic state estimation," in Proc. Int. Power Eng. Conf. (IPEC), Singapore, Dec. 2007, pp. 376-382.

[7] E. Ghahremani and I. Kamwa, "Dynamic state estimation in power system by applying the extended Kalman filter with unknown inputs to phasor measurements," IEEE Trans. Power Syst., vol. 26, no. 4 , pp. 2556-2566, Nov. 2011.

[8] A. Rouhani and A. Abur, "Tracking the machine states using a linear phasor estimator assisted dynamic state estimator," in Proc. IEEE PES General Meeting, National Harbor, MD, USA, Jul. 2014, pp. 1-5.

[9] IEEE Standard for Synchrophasor Measurements for Power Systems, IEEE Standard C37.118.1, 2011.

[10] IEEE Standard for Synchrophasor Data Transfer for Power Systems, IEEE Standard C37.118.2, 2011.

[11] S. Sarri, M. Paolone, R. Cherkaoui, A. Borghetti, F. Napolitano, and C. A. Nucci, "State estimation of active distribution networks: Comparison between WLS and iterated Kalman-filter algorithm integrating PMUs," in Proc. 3rd IEEE PES Int. Conf. Exhibit. Innov. Smart Grid Technol. (ISGT Europe), Berlin, Germany, Oct. 2012, pp. 1-8.

[12] M. Powalko, K. Rudion, P. Komarnicki, and J. Blumschein, "Observability of the distribution system," in Proc. 20th Int. Conf. Exhibit. Electr. Distrib. (CIRED), Prague, Czech Republic, Jun. 2009, pp. 1-4.

[13] D. M. Laverty, D. J. Morrow, R. J. Best, and P. A. Crossley, "Differential ROCOF relay for loss-of-mains protection of renewable generation using phasor measurement over Internet protocol," in Proc. CIGRE/IEEE Power Energy Soc. Joint Symp. Integr. Wide-Scale Renew. Res. Power Del. Syst., Calgary, AB, Canada, Jul. 2009, pp. 1-7.

[14] O. Samuelsson, M. Hemmingsson, A. H. Nielsen, K. O. H. Pedersen, and J. Rasmussen, "Monitoring of power system events at transmission and distribution level," IEEE Trans. Power Syst., vol. 21, no. 2, pp. 1007-1008, May 2006.

[15] R. J. Best, D. J. Morrow, D. M. Laverty, and P. A. Crossley, "Synchrophasor broadcast over Internet protocol for distributed generator synchronization," IEEE Trans. Power Del., vol. 25, no. 4, pp. 2835-2841, Oct. 2010.

[16] A. Carta, N. Locci, and C. Muscas, "GPS-based system for the measurement of synchronized harmonic phasors," IEEE Trans. Instrum. Meas., vol. 58, no. 3, pp. 586-593, Mar. 2009.

[17] J. Liu, J. Tang, F. Ponci, A. Monti, C. Muscas, and P. A. Pegoraro, "Trade-offs in PMU deployment for state estimation in active distribution grids," IEEE Trans. Smart Grid, vol. 3, no. 2, pp. 915-924, Jun. 2012.

[18] M. Pignati et al., "Real-time state estimation of the EPFL-campus medium-voltage grid by using PMUs," presented at the 6th Conf. Innov. Smart Grid Technol. (ISGT), Washington, DC, USA, Feb. 2014.
[19] A. von Meier, D. Culler, A. McEachern, and R. Arghandeh, "Microsynchrophasors for distribution systems," in Proc. IEEE PES 5th Innov. Smart Grid Technol. (ISGT) Conf., Washington, DC, USA, Feb. 2014, pp. $1-5$.

[20] P. Romano and M. Paolone, "Enhanced interpolated-DFT for synchrophasor estimation in FPGAs: Theory, implementation, and validation of a PMU prototype," IEEE Trans. Instrum. Meas., vol. 63, no. 12, pp. 2824-2836, Dec. 2014.

[21] K. D. Jones, J. S. Thorp, and R. M. Gardner, "Three-phase linear state estimation using phasor measurements," in Proc. IEEE PES General Meeting, Jul. 2013, pp. 1-5.

[22] K. Christakou, J.-Y. LeBoudec, M. Paolone, and D.-C. Tomozei, "Efficient computation of sensitivity coefficients of node voltages and line currents in unbalanced radial electrical distribution networks," IEEE Trans. Smart Grid, vol. 4, no. 2, pp. 741-750, Jun. 2013.

[23] G. De Carne, M. Liserre, K. Christakou, and M. Paolone, "Integrated voltage control and line congestion management in active distribution networks by means of smart transformers," in Proc. IEEE 23rd Int. Symp. Ind. Electron. (ISIE), Istanbul, Turkey, Jun. 2014, pp. 2613-2619.

[24] A. Gómez-Expósito, A. Abur, A. de la Villa Jaén, and C. Gómez-Quiles, "A multilevel state estimation paradigm for smart grids," Proc. IEEE, vol. 99, no. 6, pp. 952-976, Jun. 2011.

[25] A. R. Abbasi and A. R. Seifi, "A new coordinated approach to state estimation in integrated power systems," Int. J. Elect. Power Energy Syst., vol. 45, no. 1, pp. 152-158, Feb. 2013.

[26] H. B. Sun and B. M. Zhang, "Global state estimation for whole transmission and distribution networks," Electr. Power Syst. Res., vol. 74, no. 2, pp. 187-195, May 2005.

[27] N. Singh, E. Kliokys, H. Feldmann, R. Kussel, R. Chrustowski, and C. Joborowicz, "Power system modelling and analysis in a mixed energy management and distribution management system," IEEE Trans. Power Syst., vol. 13, no. 3, pp. 1143-1149, Aug. 1998

[28] J. Zhang, G. Welch, G. Bishop, and Z. Huang, "A two-stage Kalman filter approach for robust and real-time power system state estimation," IEEE Trans. Sustainable Energy, vol. 5, no. 2, pp. 629-636, Apr. 2014.

[29] L. Zanni, S. Sarri, M. Pignati, R. Cherkaoui, and M. Paolone, "Probabilistic assessment of the process-noise covariance matrix of discrete Kalman filter state estimation of active distribution networks," in Proc. Int. Conf. Probabilistic Methods Appl. Power Syst., Durham, U.K., Jul. 2014, pp. 1-6.

[30] W. H. Kersting, "Radial distribution test feeders," IEEE Trans. Power Syst., vol. 6, no. 3, pp. 975-985, Aug. 1991.

[31] T. Athay, R. Podmore, and S. Virmani, "A practical method for the direct analysis of transient stability," IEEE Trans. Power App. Syst., vol. 98, no. 2, pp. 573-584, Mar. 1979.

[32] N. M. Manousakis, G. N. Korres, J. N. Aliprantis, G. P. Vavourakis, and G.-C. J. Makrinas, "A two-stage state estimator for power systems with PMU and SCADA measurements," in Proc. IEEE Grenoble PowerTech (POWERTECH), Grenoble, France, Jun. 2013, pp. 1-6.

[33] S. Sarri, M. Pignati, P. Romano, L. Zanni, and M. Paolone, "A hardware-in-the-loop test platform for the performance assessment of a PMU-based real-time state estimator for active distribution networks," in Proc. PowerTech (POWERTECH), vol. 2. Eindhoven, The Netherlands, Jun./Jul. 2015, pp. 1-6.

[34] R. F. Nuqui and A. G. Phadke, "Hybrid linear state estimation utilizing synchronized phasor measurements," in Proc. IEEE Lausanne PowerTech (POWERTECH), Lausanne, Switzerland, Jul. 2007, pp. $1665-1669$.

[35] A. Jain and N. R. Shivakumar, "Power system tracking and dynamic state estimation," in Proc. IEEE/PES Power Syst. Conf. Expo., Seattle, WA, USA, Mar. 2009, pp. 1-8.

[36] X. Bian, X. R. Li, H. Chen, D. Gan, and J. Qiu, "Joint estimation of state and parameter with synchrophasors-Part I: State tracking," IEEE Trans. Power Syst., vol. 26, no. 3, pp. 1196-1208, Aug. 2011.

[37] M. Glavic and T. Van Cutsem, "Reconstructing and tracking network state from a limited number of synchrophasor measurements," IEEE Trans. Power Syst., vol. 28, no. 2, pp. 1921-1929, May 2013.

[38] C. Muscas, M. Pau, P. A. Pegoraro, S. Sulis, F. Ponci, and A. Monti, "Multiarea distribution system state estimation," IEEE Trans. Instrum. Meas., vol. 64, no. 5, pp. 1140-1148, May 2015.

[39] M. Pau, P. A. Pegoraro, and S. Sulis, "WLS distribution system state estimator based on voltages or branch-currents: Accuracy and performance comparison," in Proc. IEEE Int. Instrum. Meas. Technol. Conf., May 2013, pp. 493-498. 
[40] N. M. Manousakis, G. N. Korres, and P. S. Georgilakis, "Taxonomy of PMU placement methodologies," IEEE Trans. Power Syst., vol. 27, no. 2, pp. 1070-1077, May 2012.

[41] B. Xu and A. Abur, "Observability analysis and measurement placement for systems with PMUs," in Proc. IEEE PES Power Syst. Conf. Expo., vol. 2. New York, NY, USA, Oct. 2004, pp. 943-946.

[42] M. Paolone, A. Borghetti, and C. A. Nucci, "A synchrophasor estimation algorithm for the monitoring of active distribution networks in steady state and transient conditions," presented at the 17th Power Syst. Comput. Conf. (PSCC), Wrocław, Poland, Aug. 2014.

[43] C. Muscas, M. Pau, P. A. Pegoraro, and S. Sulis, "Effects of measurements and pseudomeasurements correlation in distribution system state estimation," IEEE Trans. Instrum. Meas., vol. 63, no. 12, pp. 2813-2823, Dec. 2014

[44] E. Caro, A. J. Conejo, and R. Mínguez, "Power system state estimation considering measurement dependencies," IEEE Trans. Power Syst., vol. 24 , no. 4 , pp. $1875-1885$, Nov. 2009.

[45] A. S. Debs and R. E. Larson, "A dynamic estimator for tracking the state of a power system," IEEE Trans. Power App. Syst., vol. 89, no. 7, pp. 1670-1678, Sep. 1970.

[46] R. E. Kalman, "A new approach to linear filtering and prediction problems," J. Basic Eng., vol. 82, no. 1, pp. 33-45, 1960

[47] G. Welch and G. Bishop, "An introduction to the Kalman filter," Dept. Comput. Sci., Univ. North Carolina, Chapel Hill, NC, USA, Tech. Rep. TR 95-041, Jul. 2006.

[48] J. Zhang, G. Welch, G. Bishop, and Z. Huang, "Reduced measurementspace dynamic state estimation (ReMeDySE) for power systems," in Proc. IEEE PES Trondheim PowerTech, Trondheim, Norway, Jun. 2011, pp. 1-7.

[49] R. K. Mehra, "On the identification of variances and adaptive Kalman filtering," IEEE Trans. Autom. Control, vol. 15, no. 2, pp. 175-184, Apr. 1970.

[50] M. Najim, Modeling, Estimation and Optimal Filtration in Signal Processing. Hoboken, NJ, USA: Wiley, 2006.

[51] B. J. Odelson, M. R. Rajamani, and J. B. Rawlings, "A new autocovariance least-squares method for estimating noise covariances," Automatica, vol. 42, no. 2, pp. 303-308, 2006.

[52] V. Krishnan, Nonlinear Filtering and Smoothing: An Introduction to Martingales, Stochastic Integrals and Estimation. New York, NY, USA: Dover, 2005.

[53] Instrument Transformers-Part 1: General Requirements, IEC Standard 61869-1, Geneva, Switzerland, 2010.

[54] Instrument Transformers-Part 2: Additional Requirements for Current Transformers, IEC Standard 61869-2, 2014.

[55] Instrument Transformers-Part 3: Additional Requirements for Inductive Voltage Transformers, IEC Standard 61869-3, 2011.

[56] J.-Y. Le Boudec, Performance Evaluation of Computer and Communication Systems. Lausanne, Switzerland: EPFL Press, 2010.

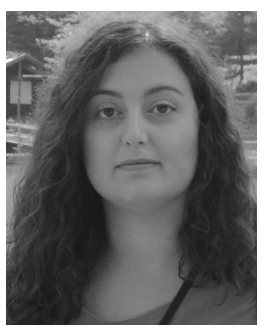

Styliani Sarri (M'12) was born in Larissa, Greece, in 1986. She received the Diploma degree from the Electrical and Computer Engineering Department, Aristotle University of Thessaloniki, Thessaloniki, Greece, in 2011, and the Ph.D. degree from the École Polytechnique Fédérale de Lausanne, Lausanne, Switzerland, in 2015.

Her current research interests include real-time electrical network monitoring and control with particular focus on state estimation of active distribution networks, real-time operation of electrical networks, and development of real-time models.

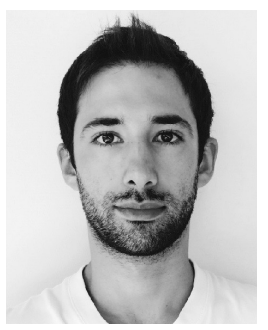

Lorenzo Zanni (M'13) was born in Italy in 1988 $\mathrm{He}$ received the B.Sc. and M.Sc. (Hons.) degrees in electrical engineering from the University of Bologna, Bologna, Italy, in 2010 and 2012, respectively. He is currently pursuing the Ph.D. degree with the Distributed Electrical System Laboratory, École Polytechnique Fédérale de Lausanne, Lausanne, Switzerland

His current research interests include real-time monitoring and control of active distribution networks with particular focus on state estimation using phasor measurement units, and synchrophasor-based fault location.

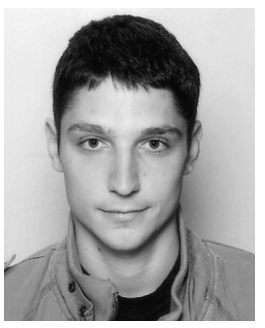

Miroslav Popovic grew up in Belgrade, Serbia, where he finished the Mathematical High School and the School of Electrical Engineering University of Belgrade, Belgrade. $\mathrm{He}$ received the B.Sc. and M.Sc. degrees in computer science from the École Polytechnique Fédérale de Lausanne, Switzerland. He is currently pursuing the $\mathrm{Ph} . \mathrm{D}$. degree under the supervision of Prof. J.-Y. Le Boudec.

His current research interests include various aspects of smart-grid communication networks.

He co-authored three papers that won the best paper awards at e-Energy 2011, CoNEXT 2012, and the IEEE World Conference on Factory Communication Systems 2015.

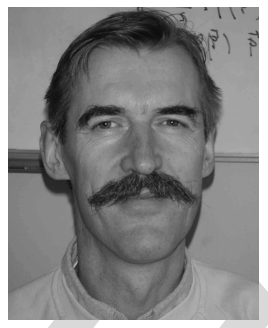

Jean-Yves Le Boudec (F'04) received the Aggregation degree in mathematics from the École Normale Supérieure de Saint-Cloud, Paris, France, in 1980, and the Ph.D. degree from the University of Rennes, Rennes, France, in 1984.

$\mathrm{He}$ was with INSA/IRISA, Rennes, from 1984 to 1987. In 1987, he joined the Network and Product Traffic Design Department, Bell Northern Research, Ottawa, ON, Canada, as a member of the Scientific Staff. In 1984, he developed analytical models of multiprocessor and multiple bus computers. In 1988, he joined the IBM Zurich Research Laboratory, Zurich, Switzerland, where he was the Manager of the Customer Premises Network Department. In 1990, he invented the concept called MAC emulation, which later became the ATM forum LAN emulation project, and developed the first ATM control point based on OSPF. He also launched public domain software for the interworking of ATM and TCP/IP under Linux. In 1994, he became a Professor with the École Polytechnique Fédérale de Lausanne (EPFL), Lausanne, Switzerland, where he is currently a Full Professor. In 1998, he proposed the first solution to the failure propagation that arises from common infrastructures in the Internet. He contributed to network calculus, a recent set of developments that forms a foundation to many traffic control concepts in the Internet, and co-authored a book on this topic. He has authored the book entitled Performance Evaluation (2010) and co-authored a textbook on information sciences for beginners. His current research interests include the performance and architecture of communication systems and smart grids.

Dr. Le Boudec received the IEEE Millennium Medal, the Infocom 2005 Best Paper Award, the CommSoc 2008 William R. Bennett Prize, the 2009 ACM Sigmetrics Best Paper Award, the ACM Conext Best Paper Award, and the 2013 EPFL I\&C Best Teacher Award. He is or has been on the Program Committee or Editorial Board of many conferences and journals, including e-Energy, Sigcomm, Sigmetrics, Infocom, Performance Evaluation, the IEEE TRANSACTIONS ON NETWORKING/ACM Transactions on Networking, and Sustainable Energy, Grids and Networks.

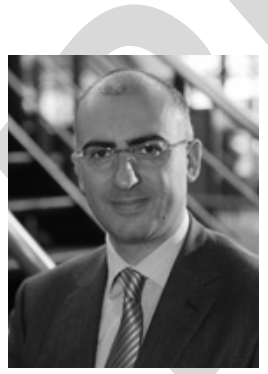

Mario Paolone (SM'10) received the M.Sc. (Hons.) and $\mathrm{Ph} . \mathrm{D}$. degrees in electrical engineering from the University of Bologna, Bologna, Italy, in 1998 and 2002, respectively.

He was appointed as an Assistant Professor of Power Systems with the University of Bologna in 2005, where he was with the Power Systems Laboratory until 2011. He is currently an Associate Professor with the École Polytechnique Fédérale de Lausanne, Switzerland, where he was the EOS Holding Chair of the Distributed Electrical Systems Laboratory. He has authored or co-authored over 200 scientific papers in reviewed journals and international conferences. His current research interests include power systems with particular reference to real-time monitoring and operation, power system protections, power systems dynamics, and power system transients.

Dr. Paolone received the Associate Professor eligibility from the Politecnico di Milano, Milan, Italy, in 2010. In 2013, he was a recipient of the IEEE Electromagnetic Compatibility Society Technical Achievement Award. He was the Co-Chair of the Technical Program Committees of the Ninth Edition of the International Conference of Power Systems Transients in 2009 and the Power Systems Computation Conference in 2016. He is the Editor-in-Chief of the Sustainable Energy, Grids and Networks (Elsevier) journal. 